\title{
Histone Demethylase UTX Compromises Articular Chondrocyte Anabolism and Aggravates Osteoarthritic Degeneration
}

\section{Wei-Shiung Lian}

Kaohsiung Chang Gung Memorial Hospital

\section{Re-Wen Wu}

Kaohsiung Chang Gung Memorial Hospital

Jih-Yang Ko

Kaohsiung Chang Gung Memorial Hospital

\section{Yu-Shan Chen}

Kaohsiung Chang Gung Memorial Hospital

\section{Shao-Yu Wang}

Kaohsiung Chang Gung Memorial Hospital

\section{Chun-Ping Yu}

Academia Sinica https://orcid.org/0000-0002-6806-9157

Holger Jahr

University Hospital RWTH Aachen, Germany

Feng-Sheng Wang ( $\square$ wangfs@ms33.hinet.net)

Kaohsiung Chang Gung Memorial Hospital https://orcid.org/0000-0001-6025-6073

\section{Article}

Keywords: UTX, H3K27 trimethylation, PRC2, chondrocytes, osteoarthritis

Posted Date: May 18th, 2021

DOI: https://doi.org/10.21203/rs.3.rs-467396/v1

License: (1) (1) This work is licensed under a Creative Commons Attribution 4.0 International License. Read Full License 


\section{Abstract}

Histone demethylase UTX removes repressive trimethyl groups at lysine 27 of histone 3 (H3K27me3) to regulate tissue integrity, while its role was not yet studied in articulating joint tissues in situ. We now found that UTX expression in articular chondrocytes positively correlated with human osteoarthritis. Utx overexpression induced chondrocyte dysfunction, cartilage degeneration and osteophyte induction in mice. In contrast, chondrocyte-specific Utx knockout in mice promoted gross articular morphology and delayed age- and collagenase-induced cartilage erosion, synovitis and osteophyte formation and largely eliminated disease-associated joint pain. Additionally, pharmacological inhibition of Utx through GSK-J4 preserved cartilage integrity. Our study is the first to suggest that Utx loss-mediated cartilage protection involved a dysregulation of polycomb repressive complex 2 core components EZH2, EED, and SUZ12 to induce H3K27 hypomethylation and a net anabolic effect. Specifically, Utx loss-of-function appears to involve, among others, Wnt10a signaling to reduce chondrocytic activities and an IGF-2-mediated stimulation of extracellular matrix synthesis.

\section{Introduction}

Osteoarthritis $(\mathrm{OA})$, characterized by a degenerative loss of articular cartilage, is the most common form of arthritis, causing joint pain, deformity, and disability in the elderly ${ }^{1}$. Severe degeneration of the cartilage extracellular matrix (ECM) causes a plethora of osteoarthritic symptoms, including synovial swelling, osteophyte formation, and subchondral plate sclerosis. Maintaining articular chondrocyte homeostasis is thus important to ensure articular tissue integrity and to protect the underlying bone from weight bearing and athletic impact on joints ${ }^{1}$. Expanding evidence suggests that dysregulated expression of, among others, chondrocyte key transcription factor SRY-box9 (SOX9), and canonical Wnt/ $\beta$-catenin signaling induce chondrocyte dysfunction which in turn accelerates $\mathrm{OA}$ development ${ }^{2-5}$. The underlying mechanisms leading to this change in metabolic activity in osteoarthritic chondrocytes remains, however, poorly elucidated.

Epigenetic pathways chemically modify DNA-bound histones or DNA nucleotides directly, like CpG dinucleotides, to control transcription through altering promoter activities ${ }^{6}$. Of these modifications, histone methylation causes chromatin condensation that represses gene expression to change biological activities $^{6}$. Recent studies show that the histone methylation status of chondrocytes plays an important role in cartilage disorders. Hypomethylation of histone 3 at lysine 36 (H3K36) correlates with chondroblastomas ${ }^{7}$, while H3K9 methylation suppresses SOX9 transcription, slowing down chondrogenesis and skeletal morphogenesis in mice lacking AT-rich interactive domain $5 b^{8}$. Moreover, aging-induced H3K4 methylation enhances cartilage loss in mice ${ }^{9}$ and loss of H3K36 methyltransferase disruptor of telomeric silencing 1-like (DOTIL) in chondrocytes results in defective murine bone development and is further relevant to human $\mathrm{OA}^{10}$. 
Lysine (K)-specific histone demethylase 6A (UTX, KDM6A) and Jumonji domain containing 3 (Jmjd3) remove trimethyl groups from histone H3K27. In contrast, histone methyltransferase polycomb repression complex 2 (PRC2) core components, including enhancer of zeste homolog 2 (EZH2), embryonic ectoderm development (EED) and PRC 2 subunit (SUZ12), catalyze trimethylation of H3K2 $27^{11}$. While UTX generally promotes gene activation and appears essential during normal development and tissue-specific differentiation ${ }^{12}$, increased $\mathrm{H} 3 \mathrm{~K} 27$ trimethylation (H3K27me3) in cartilage correlates with human hip $\mathrm{OA}^{13}$. Mice deficient in EZH1 and EZH2 in chondrocytes display poor chondrogenesis and skeletal tissue underdevelopment along with low H3K27me3 abundance ${ }^{14}$. Interestingly, EZH2 deletion accelerates the development of $\mathrm{OA}^{15}$, while chondrocyte-specific EED knockout mice show a deformed skeleton and decreased chondrocyte survival ${ }^{16}$. Recently, JMJD3 is shown to regulate in vitro chondrogenic differentiation of human mesenchymal stem cells in monolayer culture ${ }^{17}$. The role of UTX during articular cartilage homeostasis and progression of $\mathrm{OA}$ remains poorly understood.

This study aimed at elucidating how UTX activity affected H3K27 methylation state and how this subsequently altered the articular cartilage phenotype. To this end, we used human OA specimens, RNA interference and pharmacological inhibition in chondrocytes as well as chondrocyte-specific UTX knockout mice to study genome-wide chondrocyte-specific maker expression. Our analyses revealed that UTX loss in primary chondrocytes suppressed PRC2-mediated H3K27 trimethylation in promoter regions of key regulators of the chondrocytic phenotype, thereby facilitating transcription of Igf-2, but suppressing that of Wnt10a, to promote cartilage metabolism and suppress OA progression.

\section{Results}

\section{UTX and H3K27 trimethyl marks correlates with human gonarthritis}

Safranin-0-stained sections showed typical histopathology alterations in OA cartilage as compared to macroscopically normal, non-OA regions in the knee. UTX expression was significantly increased in OA as compared to non-OA groups (Fig. 1a). Osteoarthritic chondrocytes further exhibited strong UTX (Fig. 1b) and H3K27me3 (Fig. 1C) immunostaining, respectively, confirming this on protein level.

\section{UTX inhibits ECM production in chondrocytes}

Next, we investigated Utx expression in relation to ECM expression in articular chondrocytes.

Chondrocytes from knee joints of 7-day-old mice were transfected with Utx expressing vectors or Utx siRNAs, respectively. Forced Utx expression increased H3K27me3 levels, but decreased Sox9 abundance (Fig. 1d). Chromatin immunoprecipitation (ChIP)-PCR analysis verified that forced Utx expression promoted H3K27me3 enrichment at the proximal Sox9 promoter region (Fig. 1e) and reduced expression of Sox9 and cartilage-specific key ECM markers Col2a1 and Acan (Fig. 1f). Consequently, Alcian blue staining confirmed ECM loss in micro-mass cultures as compared to scrambled controls (Fig. 1g). On the contrary, silencing Utx expression by RNAi reduced overall H3K27me3 level and its occupancy at the Sox9 promoter (Fig. 1d and e), but significantly enhanced both aforementioned chondrocyte markers (Fig. 1f) and ECM production as compared to scrambled controls (Fig. 1g). 


\section{Forced Utx expression causes OA-like symptoms}

Utx-mediated suppression of chondrocyte function in vitro prompted us to investigate whether increased Utx expression affected the integrity of diarthrodial joints. Tibiofemoral compartments were intraarticularly injected to lentivirally overexpress Utx (Fig. 2a). Subsequently, articular chondrocytes displayed strong Utx immunostaining (Fig. 2b). Forced Utx expression further induced signs of severe OA development, including cartilage degeneration and synovial hyperplasia (Fig. 2c) together with significant increases in OARSI- and synovitis-scores (Fig. 2d) 8 weeks after injection. Furthermore, osteophyte development was increased (Fig. 2e), while subchondral bone mineral density (BMD) and trabecular bone volume (BV/TV) were decreased (Fig. 2f) through Utx overexpression. Utx overexpressing chondrocytes further showed reduced Sox9 and Acan immunostaining, respectively, as compared to controls (Fig. $2 \mathrm{~g}$ ).

\section{Characterization of cartilage-specific Utx KO mice}

As Utx knockdown appeared to enhance expression of certain chondrocyte differentiation markers in vitro, we next aimed at generating chondrocyte-specific Utx knockout mice. Mice carrying Utx flox flanked exon 24 , which is indispensable for its demethylase activity ${ }^{18}$, were crossed with collagen type II (Col2a1)-driven Cre recombinase mice (Col2a $1^{\mathrm{cre} /+}$ ) to breed knockout mice (Col2a $\left.1^{\mathrm{cre} /{ }_{-}-\mathrm{Utx}}{ }^{\mathrm{fl} / \mathrm{fl}} ; \mathrm{KO}\right)$. Genotyping subsequently confirmed cartilage-specific absence of Utx expression in these animals (supplementary Fig. S1). Birth frequency of homozygous KO mice was $20 \%$ and thus similar to the expected Mendelian frequency. Appearance, hair color, and serum biochemistry profiles of KO mice were

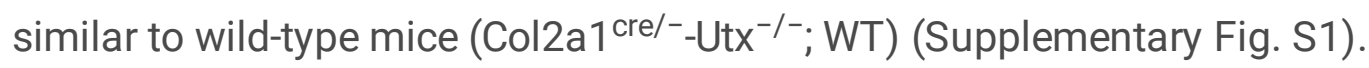

Upon confirmation of absence of Utx mRNA and protein expression (Fig. 3a), respectively, H3K27me3 level was reduced in chondrocytes (Fig. 3b). Consistent with earlier in vitro analyses, articular Utx KOchondrocytes did not show Utx, or convincing H3K27me3, immunostaining (Fig. 3c). Jmjd3 mRNA expression and protein level and immunostaining, respectively, was not significantly altered in chondrocytes from these Utx KO mice (Supplementary Fig. S2). Whole mount staining of 7-day-old specimens revealed that macroscopic phenotype of skeleton in Utx KO mice was like WT mice (Fig. 3d). Importantly, femur and tibia length were significantly increased in Utx KO mice. Body weight was not significantly affected at all tested timepoint (Fig. 3e).

\section{UTX deletion protects articular cartilage integrity}

Gross articular morphology of Utx KO mice was screened over a time course of up to 9 months by Safranin-O staining (Fig. 3f) to evaluate cartilage matrix quality, where the intensity of the staining is proportional to the proteoglycan content of the tissue. Cartilage erosion (Fig. 3f) together with osteophyte formation (Fig. $3 g$ ) was evident in articular cartilage gross morphology at 9 months, with a $66 \%$ reduction in total osteophyte volume in Utx KO animals as compared to the WT (Fig. 3h). Overall, Utx KO mice showed the better articular cartilage with significantly lower OARSI scores at 9 months of age (Fig. $3 \mathrm{~h}$ ).

The thickness of articular cartilage almost doubled between 2 and 4 weeks of age in both types of animals, to become almost stagnant between 4 and 16 weeks. As compared to WT animals, Utx KO mice 
have relatively thicker articular cartilage at all tested timepoints (Fig. 3i). However, 9 months old animals in both groups showed a, possibly age-dependent, thinning of the articular cartilage, with that of Utx KO mice remaining relatively thicker than that of WT animals (Fig. 3i). In addition, the uncalcified portion of the articular cartilage remained relative thicker in Utx KO mice as compared to WT, too (Fig. 3j). While the number of chondrocytes in articular cartilage declined age-dependently steadily in both groups (Fig. 3k), their numbers stayed always relatively higher in age-matched Utx KO animals. Interestingly, in 9 months old animals the calcified zone was relatively thicker in Utx KO animals (Fig. 3l). Furthermore, articular cartilage of 16 weeks old Utx KO mice showed relatively stronger Sox9, Acan and Col2a1 immunostaining (Fig. $3 \mathrm{~m}$ ) as compared to WT, respectively, indicating better ECM homeostasis and an improved potential to retard $\mathrm{OA}$ in Utx $\mathrm{KO}$ animals.

\section{UTX knockout retarded OA progression and improves pain scores}

Given that high Utx expression was associated with human knee OA (Fig. 1) and articular cartilage degeneration in mice (Fig. 2), we used intra-articular collagenase injections as an accepted model to induce OA development in vivo ${ }^{19,20}$. In WT mice, collagenase-injected joints showed severe cartilage loss (Fig. 4a) and increased OARSI and synovitis scores (Fig. 4b), respectively, at 8 weeks after injections. Additionally, osteophytes were more prominently developed in WT than in Utx KO animals, revealing increased overall volumes by quantitative $\mu C T$ analyses upon OA induction in WT animals (Fig. 4c). Subchondral BMD and BV/TV were significantly decreased in the injured joints (Fig. 4d). Of note, cartilage destruction, osteophyte formation, and subchondral bone loss was all less severe in Utx KO mice.

Pain and deregulated joint kinematics are prominent characteristic of $O A$ and the former is a primary symptom of $O A$ in humans and the leading cause of disability ${ }^{21}$. We thus next conducted catwalk analyses as a well-accepted model ${ }^{22}$ to assess pain and gait properties resulting from body-weight loading in animals of collagenase-treated and control legs. WT mice with OA knees showed deviated body axis (Supplementary Fig. S3) and irregular footprint (Fig. 4e) and gait profiles, including decreased footprint area, maximum contact area, duration of stand, and duty cycles (Fig. 4f). Mobility parameters, like moving time and distance were also significantly reduced as evident from open field assessment (Supplementary Fig. S3). In contrast, walking patterns, body posture and mobility of collagenase-injected Utx KO animals were significantly improved, which indicates that Utx loss of function alleviates joint pain in this model. Likewise, Utx KO mice kept showing a prominent Sox9 and aggrecan, but a weak H3K27me3, immunoreactivity in chondrocytes within collagenase-treated articular tissue (Fig. 4g).

\section{UTX inhibition attenuates OA progression}

In addition, one week after collagenase injection, Utx inhibitor GSK-J4 was intra-articularly injected to screen for potential OA-modifying effects. Consistently, this treatment significantly reduced cartilage loss and synovitis (Fig. 5a) together with decreases in OARSI and synovitis scores (Fig. 5b) in collagenaseinjected joints. GSK-J4-treament further reduced osteophyte formation (Fig. $5 \mathrm{c}$ ) and subchondral bone loss (Fig. 5d), respectively. The irregular footprint (Fig. 5e) and gait profiles (Fig. 5f) from osteoarthritic 
joints were also partially reversed upon GSK-J4 injection. The treatment further revealed improved Sox 9 and aggrecan immunostaining, respectively, but reduced H3K27me3 immunoreactivity (Fig. 5g).

\section{UTX loss decreases H3K27me3 enrichment at cartilage marker genes}

We conducted genome-wide ChIP-Seq analyses to identify changes in the H3K27me3 binding epigenomic landscape that might have contributed to the improved chondrocyte marker gene expression in Utx KO mice. Of $19531 \mathrm{H} 3 \mathrm{~K} 27 \mathrm{me} 3$ binding sites, $91.7 \%$ and $7.2 \%$ were enriched in genomes of WT and Utx KO animals, respectively, (Fig. 6a). Loss of histone eraser Utx globally decreased the H3K27me3 occupancy in promoters preferably decreased within a $5 \mathrm{~kb}$ up- and downstream region of the respective transcriptional start sites (Fig. 6b), which consequently resulted in a higher normalized transcriptional expression (RPKM) in WT chondrocytes (Fig. 6c). The enrichment of H3K27me3 in exon region was increased in $\mathrm{KO}$ mice, which was indicative of gene transcription alteration upon Utx deletion (Fig. 6d). Pathway ontology analyses suggested that Utx knockout facilitated cellular processes related to cartilage and skeletal development, including ossification and bone mineralization, as well as enhanced inhibition of cellular senescence (Fig. 6e). Gene set enrichment analysis furthermore indicated transcription of Igf-2 (Fig. 6f) and Wnt10a (Fig. 6g) together with significant increases in Dkk1, sFRP1, Igf-R2, Wnt4, and Wnt7a expression in Utx KO mice (Supplementary Fig. S4).

From the H3K27me3 repressive signatures, Igf-2 and Wnt10a but also Sox 9 were selected for subsequent experiments as they are known to regulate chondrocyte function and cartilage development ${ }^{23,24}$. Figure 7a shows the spatial distribution of H3K27me3 bindig sites in the coding sequence of Igf-2, Wnt10a, and Sox9, respectively, also indicating the relative position of the respective TSS, next to a quantified representation of the relative H3K27me3 occupancy per gene (Fig. 7b). Upon Utx loss, mRNA expression of Igf-2 and Sox 9 was consequently increased, while that of Wnt10a was significantly reduced (Fig. 7c).

Expression of Sox9, Col2a1 and Acan (Fig. 7d, IgG control) and glycosaminoglycan synthesis (Fig. 7e) were much higher in Utx KO cells than in WT cells. These effects were significantly suppressed upon Igf-2 antibody administration. Also, forced Wnt10a expression suppressed these cartilage marker genes (Fig. 7d) and ECM production (Fig. 7e) in Utx KO chondrocytes. Our data suggest that Igf-2 positively stimulates chondrocyte anabolism, whereas Wnt10a-mediated signaling seems rather deleterious to cartilage ECM synthesis.

\section{UTX loss-mediated H3K27 hypomethylation involves PRC2}

To understand how a deficiency in histone eraser Utx caused a seemingly contradictory H3K27 hypomethylation, we investigated whether PRC2 signaling was changed in chondrocytes. Increased Ezh2 but reduced Eed, Suz12, and H3K27me3 abundances was evident in Utx KO cells (Fig. 8a). Additionally, Ezh2 RNAi decreased H3K27me3 levels (Fig. 8b) along with a significant decrease in H3K27me3 enrichment at the Sox9 promoter region (Fig. 8c), resulting in an upregulation of chondrocyte markers (Fig. 8d) and ECM production (Fig. 8e), respectively, in Utx KO chondrocytes. On the contrary, forced Eed and Suz12 expression increased H3K27me3 abundance (Fig. 8f) and the H3K27me3 occupancy at the 
Sox9 promoter in particular, relative to WT cells (Fig. 8g). This subsequently repressed chondrocyte markers (Fig. 8h) and ECM synthesis (Fig. 8i). Experimental results suggested that loss-of-function of Utx deregulated PRC2 action, maintaining H3K27 relatively hypomethylated and therefore enhanced chondrocytic marker gene expression. Low Eed or Suz12 abundance appeared to inhibit Eeh2 and its ability to trimethylate H3K27.

\section{Discussion}

Epigenetic changes occur during $\mathrm{OA}$ and histone methylation, in particular, appear to imbalance chondrocyte metabolism ${ }^{25,26}$. Our data now associate expression of histone eraser UTX with OA development in humans and correlate UTX and H3K27 trimethylation in articular cartilage with chondrocyte marker gene expression in vitro. This is in agreement with the only other study demonstrating high H3K27me3 abundances in human hip osteoarthritic cartilage ${ }^{14}$. We further, for the first time, manipulated Utx activity through RNAi and lentiviral overexpression, established a murine Utx $\mathrm{KO}$ model and evaluated intraarticular effects of local Utx-specific inhibition in vivo. Our comprehensive study reveals a novel non-canonical role of the H3K27me2/3-specific demethylase Utx, in concert with PRC2 core components, in articular chondrocytes homeostasis and the progression of OA.

We used Chip-Seq to screen global changes in gene expression between WT and Utx KO chondrocytes to identify molecular mechanisms protecting cartilage against degradation. GO terms already revealed major expressional changes occurring in gene regulation networks related to mineralization, ossification and senescence. Candidates like Sox9, Igf-2 and Wnt10a arising from enrichment scores were then further analyzed to reveal an aberrant H3K27me3 occupation at their respective promoter loci between WT and Utx KO chromatin. IGF-2 and Wnt signaling components were known to regulate chondrocyte function and cartilage development ${ }^{23,24}$. Our enrichment scores now revealed upregulation of many markers associated with a proper articular chondrocyte phenotype, like relevant collagens of the cartilage ECM (e.g., Col9a2) or the cartilage-specific acidic protein 1 (CRTAC1) ${ }^{27}$ and that of BMP antagonists like GREM1. Col9a2 is considered to be a chondrocyte-specific marker gene, in addition to Col2a1, Col11a2 and aggrecan ${ }^{28}$.

Sox9 is a prominent activator of Col2a 1 transcription, making it a master regulator of the chondrocyte phenotype ${ }^{29}$. To show a Utx-dependent compromised chondrocytic activity, we used key chondrocyte marker genes Sox9, Col2a1 and Acan as readouts, and quantified proteoglycan production as a measure of ECM quality. Using UTX-specific inhibitor GSK-J4, shown to inhibit H3K27 demethylation of UTX target genes ${ }^{30}$, and confirmed anabolic effects on ECM level in vitro and being in line with the observed overall improved relative thickness of the articular cartilage in vivo. BMP signaling ultimately results in unwanted hypertrophy in articular chondrocytes. Leijten et al. already showed that decreased GREM1 and DKK1 gene expression in cartilage were correlated with $O A^{31}$. Harmonization of BMP and WNT signaling are important to maintain articular cartilage integrity. While BMPs ${ }^{32}$ and canonical WNTs ${ }^{33}$ may exert prohypertrophic actions under certain conditions, the parallel upregulation of BMP antagonists and WNT 
antagonists, like sFRP-1 or DKK1, may thus explain the overall chondroprotective net effect of Utx loss in vitro and in vivo.

Interestingly, adult articular cartilage has been long considered a post-mitotic tissue with terminally differentiated chondrocytes ${ }^{34,35}$, but this textbook dogma has been questioned recently. A 'phenotypic plasticity' of articular chondrocytes has then been associated with $\mathrm{OA}^{36}$ in which chondrocytes dedifferentiation towards a more fibroblast-like phenotype ${ }^{37}$. To this end, Wnt signaling is an important regulator of cell plasticity ${ }^{38}$ and the Wnt-frizzled- $\beta$-catenin pathway appears activated in $0 A^{39,40}$. Expression of Wnt4 and Wnt10, in particular, can stimulate osteogenesis ${ }^{41}$, and excessive Wnt signaling can also lead to increased osteophyte formation ${ }^{42}$, which is in agreement with our findings. Recently, mouse genetic analysis also revealed that $\mathrm{Wnt} / \beta$-catenin signaling components are important to regulate Sox 9 and Runx 2 and Wnt signaling can thus suppress chondrogenic differentiation, while increasing osteoblastic differentiation ${ }^{43}$. In line with our hypothesis, H3K27me3 demethylase UTX has earlier been shown to be essential to tissue development and stem cell plasticity ${ }^{44}$. In contrast, Wnt inhibition attenuates $\mathrm{OA}$ development through anti-catabolic and anti-fibrotic effects on chondrocytes and synovial fibroblasts, respectively ${ }^{45}$. Wnt signaling plays a context-dependent role in the development of OA and upregulated expression of canonical Wnts, like Wnt10, and non-canonical, like Wnt4 and Wnt7 ${ }^{46}$, together with Wnt inhibitors like sFRPs and Dkks hint towards a delicate balance of this system.

Currently developed Wnt inhibitors as disease-modifying osteoarthritis drugs (DMOAD) ${ }^{40}$ underscore their potential. Local intraarticular modulation of this pathway through Utx inhibition is another effective means to change the course of this joint disease. To the best of our knowledge, no further information on a direct involvement of Utx in regulating the IGF cell signaling axis in OA exists, while IGF-2 is known to regulate cartilage development ${ }^{47}$. IGF-2 also compromises ECM underproduction in inflamed chondrocytes and preserves cartilage integrity even in a model of experimental osteoarthritis ${ }^{48}$. This may, partly, explain the beneficial effects of Utx KO on ECM synthesis in chondrocytes. However, two groups independently reported that homologue Utx-1 in C. elegans regulates its life span through targeting IGF-1 pathway ${ }^{49,50}$. This may link age-related changes in Utx activity to age-related chondrocyte senescence and onset of primary $\mathrm{OA}^{2}$. IGF signaling also regulates cell proliferation, differentiation and apoptosis in cartilage ${ }^{51}$. While IGF signaling is considered a promising drug target, complex negative feedback regulation and the systemic importance of glucose homeostasis helps explaining the failures of single target therapies aiming at regulating IGF signaling ${ }^{51}$. Modulating IGF signaling indirectly and intraarticularly through pharmacological Utx modulation may thus be more promising.

Utx knockout largely protected against signs of cartilage degeneration in primary OA and in an experimental murine model of induced secondary OA development. We then used a pharmacological Utx inhibitor in the latter model to demonstrate protection against gonarthrotic changes in the joint, confirming anabolic effects on ECM level in vitro and being in line with the observed overall improved relative thickness of the articular cartilage in vivo. Pain is one of prominent symptoms of $\mathrm{OA}^{52}$. We thus 
used catwalk analyses as a well-accepted model to assess pain in rodents ${ }^{53}$, like that resulting from joint tissue degeneration due to deregulated joint kinematics ${ }^{20}$ in collagenase-treated limbs. Upon intraarticular injection, Utx inhibitor GSK-J4 not only protected against joint tissue degradation, but also against pain. The latter was evident from derived from restoration of normal gait profiles and mobility in animals with injured joints. To the best of our knowledge, only a single other study very recently studied Utx in chondrocytes ex vivo, also using GSK-J4 ${ }^{17}$. These earlier results also hinting towards a cartilageprotective effect of Utx inhibition, but it is well accepted that the complex TGF- $\beta$ signaling in adult cartilage in vivo cannot be fully appreciated by in vitro models of chondrogenesis used in that study.

UTX, a member of the Jumonji C family of histone erasers, usually removes di- and tri-methyl groups on H3K27 to promote target gene activation ${ }^{54}$. Surprisingly, loss-of-function of Utx now activated chondroprotective pathways and mRNA expression of Igf2 and Sox9 in particular, while that of Wnt10a was significantly reduced (Fig. 7). Additionally, Utx KO contra-intuitively reduced the H3K27me3 occupation at the promoter loci of these genes. Apparently, other regulatory pathways potentially contribute and UTX loss can indeed enhance the EZH2-induced H3K27 trimethylation ${ }^{55}$. We thus postulate that PRC2 core components participate in the UTX deletion-induced H3K27 hypomethylation in chondrocytes. Our data now reveal that upon UTX loss, EZH2 appears to curtail chondrocytic metabolism as EZH2 RNAi-mediated suppression maintained H3K27 hypomethylated and improves ECM synthesis, whereas downregulation of EED or SUZ12 appears to stimulate matrix anabolism, as restoring EED or SUZ12 results in H3K27 hypermethylation and compromised ECM synthesis. In agreement with our data, chondrocyte-specific EZH2 or EED knockout mice show poor cartilage development and defective bone growth ${ }^{13,15}$. EED thus appears functionally indispensable for trimethylation of $\mathrm{H} 3 \mathrm{~K} 27$ by $\mathrm{EZH} 2^{56}$. Our study thus revealed a new paradigm in which opposing action of PRC2 components are responsible for UTX loss-mediated H3K27 hypomethylation to maintain proper ECM homeostasis in cartilage. Weak EED and SUZ12 signaling blocked EZH2-mediated H3K27 trimethylation, driving chondrocytes in KO mice to produce abundant extracellular matrices.

Utx is further a member of MLL2 H3K4 methyltransferase complex and got additional demethylase independent roles in chromatin remodeling through an interaction with SWI/SNF complex ${ }^{57,58}$. While activation of non-canonical Wnt signaling may promote osteogenic differentiation through $\mathrm{H} 3 \mathrm{~K} 9$ methylation, WNT10A facilitated $\beta$-catenin stabilization potentially also causes cartilage mineralization. In mammals, PRC2 core components EZH2 and EZH1 are important for writing trimethylation of $\mathrm{H} 3 \mathrm{~K} 27^{59}$. Of note, H3K27 methyltransferase EZH2 is known to repress Wnt signaling components ${ }^{60}$. PRC2 and H3K27me3 are involved in bivalent control of transcription activation and repression during stem cell fate commitment ${ }^{61}$, in line with the earlier discussed plasticity of chondrocytes in OA. To this end, our data is in agreement with studies demonstrating that UTX KO decreases H3K27 trimethylation to alter mesenchymal stem cells differentiation ${ }^{62}$. Although histone demethylase Jmjd3 is found to remove trimethyl group of H3K27 in various cell types ${ }^{11}$, its expression in chondrocytes was not changed in UTX $\mathrm{KO}$ mice. In general, Polycomb-group proteins together with their target genes control differentiation program in a dynamic manner. Co-localization of PRC2 with H3K27me3 is required to catalyze 
trimethylation ${ }^{61}$. As Polycomb-group proteins regulate gene silencing, repressing transdifferentiation in a H3K27me3 dependent manner ${ }^{63}$ and the latter appears to be the link between inflammation and reprogramming of the epigenome ${ }^{63}$, this may - at least partly - explain our findings.

In conclusion, loss of function of Utx appears to be chondroprotective. However, a seemingly contradictory trimethylation status was observed at the selected gene loci of key chondrocyte markers: loss of histone eraser Utx caused a depletion of H3K27me3 occupation at these domains. To this end, we identified a novel interaction between Utx and PRC2 core complex components. Our current model of the epigenetic regulation of cartilage metabolism is illustrated in Fig. 9; on bivalent loci, PRC2 activity acts in concert with Utx to either stimulate cartilage anabolism or activate phenotypic de-differentiation and ECM deterioration through activating Wnt signaling.

Our study sheds new light onto epigenetic causes of OA development. A better understanding of the molecular composition, but also of the interactions between, the different canonical and non-canonical PRC complexes with histone erasers of the Jumonji family is still needed. Altogether, the complexity of biological functions assigned to Utx in general, and in cartilage in particular, has just started to emerge. We showed, for the first time, in a multimodal approach using human samples and animal models as well as RNAi and pharmacological intervention that targeted manipulation of Utx activity appears to hold a lot of potential for the development of future anti-OA therapies. While repetitive intraarticular injections with these inhibitors may be required to treat chronic diseases like OA, biomaterial-based controlled delivery systems may be a realistic clinical treatment option.

\section{Methods}

\section{Human knee biopsies}

Protocols for harvesting clinical specimens were in compliance with the local ethical guidelines and approved by Chang Gung Medical Foundation Institutional Review Board (IRB affidavit number: 1045248B and 106-2251C). Thirty-four patients inflicted with radiographic signs of end-stage knee OA were included. Patients were fully informed and written consent obtained. During total knee arthroplasty, tissue specimens were harvested from osteoarthritic regions and macroscopic healthy regions in the joint, lateral to the injured site (diagnosed by two orthopedic surgeons). Subsequent analyses were performed by investigators blinded to the grouping.

\section{Chondrocyte isolation and in vitro cultures}

All breeding programs and experimental protocols for laboratory animals were in compliance with guidelines of animal wellbeing and approved by the Institutional Animal Care and Use Committee of Kaohsiung Chang Gung Memorial Hospital (IACUC Affidavit No. 2017112701). Rodents had ad libitum access to chow and drinking water. Seven-day-old male C57BL/ 6 mice were euthanatized, chondrocytes were isolated from knee joints and incubated in DMEM with $10 \%$ fetal bovine serum, as previously described $^{64}$. For micro-mass cultures, $5 \times 10^{5}$ cells were suspended in $10 \mu$ l of medium and gently 
pipetted to form a drop onto a culture plate prior to incubation for 7 days in a $37 \mathrm{C}$ humidified incubator. ECM synthesis was detected by Alcian blue staining, with the stain subsequently dissolved with $50 \mu \mathrm{l}$ of 6 $M$ guanidine hydrochloride. The extracts were quantified at $620 \mathrm{~nm}$ in a spectrophotometer and normalized to total cellular protein. In some experiments, chondrocytes were incubated in basal medium containing $10 \mathrm{ng} / \mathrm{ml} \mathrm{IGF-2} \mathrm{antibody} \mathrm{(R \& D} \mathrm{Systems)} \mathrm{or} 10 \mathrm{ng} / \mathrm{ml} \mathrm{IgG} \mathrm{for} 7$ days.

\section{Lentiviral transduction and RNAi experiments}

Vectors (pMIF-cGFP-zeo; System Biosciences) encoding full length UTX, EED, SUZ12 or Wnt10a cDNAs, respectively, were transduced together with packaging vectors PPACKF1 (System Biosciences) into 293T cells. In a subset of experiments, vectors encoding Utx (s75839) or Ezh2 (s65775) siRNAs were obtained from Thermo Fisher Scientific. After incubating for 5 days in a humidified cell culture incubator, culture supernatants were harvested and centrifuged at $10,000 \times g, 4^{\circ} \mathrm{C}$ for $30 \mathrm{~min}$. Titration of virus particle condensate was performed using LentiX RT-qPCR Titration Kits (Clontech) and then suspended to result in $10^{7}$ infectious units/ $\mu$ l. Chondrocytes $\left(5 \times 10^{5}\right.$ cells) were mixed with $100 \mathrm{ml}$ of the lentivirus particle suspension for 24 hours and suspension for 24 hours and then harvested for micro-mass cultures.

\section{Lentivirus-shuttled UTX gene transfer into knee joints}

Lentivirus suspensions ( $1 \times 10^{9} / \mu$ infectious unit) were prepared for in vivo studies. Male C57BL/ 6 mice (12 weeks old) were anesthetized, $10 \mu \mathrm{U}$ UTX or mock virus particle suspension were intra-articularly injected (Digital Stereotaxic Instruments, RWD) into left knees under guidance of an ultrasonography system with a high frequency transducer $\left(10-22 \mathrm{MHz}^{2} \mathrm{LOGIO}^{\mathrm{TM}}, \mathrm{GE}\right.$ Healthcare). At 8 weeks after injection, animals were euthanized and knee joints were dissected for $\mu \mathrm{CT}$ imaging and histological assessment.

\section{Chondrocyte-specific UTX knockout mice}

Col2a $1^{\text {cre/+}}$ C57BL/ 6 mice (Jackson Laboratory) were mated with Utx flox mice (Jackson Laboratory) to breed homozygous KO mice (Co12a ${ }^{\mathrm{Cre} /+}-\mathrm{UTX}^{\mathrm{fl} / \mathrm{fl}}$ ) and WT mice (Col2a $1^{\left.\mathrm{Cre} /{ }_{-}-\mathrm{UTX}^{-/-}\right) \text {. PureLink }}{ }^{\mathrm{TM}}$ genomic DNA Mini Kits (Invitrogen) were utilized to extract genomic DNA in tail tips. Genotypes were confirmed using primers for flox (forward: 5'-TGAACGCTTACGGAAC-3'; reverse: 5'-AAATCATGCTGGA ACCTAGAAC-3'), and Cre (forward: 5'-AGGTGACGTAATTCAGG-3'; reverse: 5'-

CAATTGCTCATATGGACATGTAC-3') and a thermal program ABI 7900 Detection System (Applied Biosystems).

\section{RT-quantitative PCR assessment of mRNA expression}

One $\mu \mathrm{g}$ of total RNA extracted from cell cultures were pipetted for reverse transcription. Twenty ng RT products were mixed with 2x TaqMan ${ }^{\circledR}$ Universal PCR Master Mix (Applied Biosystems) and $2.5 \mu \mathrm{M}$ primers for UTX, Jmjd3, Sox9, Col2a1, Acan, Igf-2, Igfr2, Wnt4, Wnt7a, Wnt10a, Dkk1, sFRP1, and 18S rRNA (Supplemental Table 1). PCR reactions were performed in an ABI 7900 Detection System. The gradient thermal program, amplification specificity, and calculation of relative mRNA expression were performed as previously described ${ }^{19}$.

\section{Histomorphometric analyses}


Intact knee joints including tibiae and femurs were dissected and fixed in 10\% PBS-buffered formaldehyde, decalcified in 10\% EDTA and then embedded in paraffin wax. Sagittal sections at the medial mid-condylar region were subjected to Safranin-O staining. Histological examination and image acquisition of proximal tibiae were performed using digital slide scanner (Pannoramic MIDI II, 3D HISTECH Ltd.), spanning anterior-to-posterior tibial areas and using six randomly selected section from each specimen in increments of $200 \mu \mathrm{m}$ for histological evaluation. Average thickness of total articular cartilage (area between articular surface and cement line), uncalcified articular cartilage (area between tidemark and articular surface), and calcified articular cartilage (area between tidemark and cement line) was measured, as previously described ${ }^{65,66}$. In some experiments, sections were stained with hematoxylin and eosin. Articular chondrocytes in each field $\left(50 \mu \mathrm{m}^{2}\right)$ were counted; and six fields in each section of each specimen were selected for study.

\section{Assessment of OA histopathology}

Ten sections of knee joints spanning $200 \mu \mathrm{m}$ were subjected to Safranin-0 staining. The severity of articular cartilage damage, like Safranin-0 staining intensity, cartilage fibrillation, and erosion was examined using Zeiss light microscope and graded, according to the guideline of Osteoarthritis Research Society International (OARSI score) ${ }^{67}$. Severity of synovitis, including cellularity and synovial membrane thickness, in the region of interest was evaluated using a $0-3$ scoring system, with $0=$ normal, $1=$ moderate, and 3 = severe degeneration.

\section{Immunohistochemistry}

After removing wax and retrieving antigen, sections were probed using primary antibodies against UTX, Jmjd3, H3K27me3, SOX9, collagen II, aggrecan, respectively, and followed by Super Sensitive ${ }^{\mathrm{TM}} \mathrm{IHC}$ Detection Systems (BioGenex Laboratories) containing non-biotin horseradish peroxidase-conjugated IgG and chromogenic substrate 3,3'-diaminobenzidine. Cell nuclei were counterstained with hematoxylin. Number of immuno-stained cells within each field $\left(50 \mu \mathrm{m}^{2}\right)$ was counted; and 3 fields in each section and 3 sections of each specimen were selected for microscopic examination.

\section{Collagenase-induced $O A$ in vivo model}

Twelve weeks old male WT and KO mice were anesthetized, left knees were intra-articularly injected with 1 unit of collagenase (Clostridium histolyticum, Aldrich-Sigma) or sterile saline ${ }^{19,20}$ under ultrasonographic guidance. Weight-bearing activity was allowed throughout the study period. At 8 weeks after collagenase injection, mice were subjected to euthanasia, knee joints, including proximal parts of femurs and tibiae, were excised for evaluating articular histopathology.

\section{Intra-articular UTX inhibitor administration}

Male C57BL/ 6 mice were anesthetized, and 1 unit of collagenase was intra-articularly injected to induce OA. Two weeks after injection, animals were randomly divided into two equally sized groups $(n=5$ or 6$)$ and affected knees either received intra-articular injections with $250 \mu \mathrm{g} / \mathrm{kg}$ UTX inhibitor GSK-J4 (Tocris) or a vehicle (DMSO). Eight weeks after the initial injection, animals were euthanatized, and knee joints dissected for further analyses. 


\section{Gait and mobility analyses}

At 8 weeks after collagenase injection, posture and gait profiles of mice with affected knees were examined using a CatWalk system (Noldus Information Technology). Each mouse was analyzed 3 times. While mice walked through the passage, images and patterns of posture, movement, and footprints on the walkway were synchronically recorded with highspeed cameras and highly-sensitive sensors of the system. Print area $\left(\mathrm{mm}^{2}\right)$, maximum contact area $\left(\mathrm{mm}^{2}\right)$, duration of stand $(\mathrm{sec})$ and duty cycle $(\mathrm{sec})$ were computed with CatWalk software 9.1 and CatWalk XT's Automatic Footprint Classification software. In some experiments, each mouse was placed in an open field area $(30 \mathrm{~cm} \times 30 \mathrm{~cm} \times 30 \mathrm{~cm})$ for $15 \mathrm{~min}$. Distance and duration of mobility were computed automatically by the system.

\section{Micro-CT Analyses}

The microstructure of mouse knee joints between metaphyseal region of proximal tibiae and proximal femurs were evaluated using a Skyscan $1176 \mu$ CT scanner (Bruker). Each isotropic $9 \mu \mathrm{m}$ voxel size X-ray scan was performed at $70 \mathrm{keV}$ and $500 \mu \mathrm{A}$ intensity for $69 \mathrm{~ms}$. Reconstruction of 400 scanned sections was performed using SKYSCAN® CT-Analyser software. Osteophyte formation, like radiopaque tissue ectopic around knee joints or protruded from skeletons, as well as trabecular morphology of subchondral bone in proximal tibiae were diagnosed, as previously described ${ }^{68}$. Osteophyte volume $\left(\mathrm{mm}^{3}\right)$, bone mineral density $\left(\mathrm{g} / \mathrm{mm}^{3}\right)$, and total bone volume (BV/TV) were quantified using the software.

\section{ChIP sequencing (ChIP-seq) of H3K27me3 binding sites}

H3K27me3 in $5 \times 10^{6}$ chondrocyte cultures were immunoprecipitated using monoclonal antibodies for H3K27me3 (ab6002; Abcam) or IgG (Millipore). Chromatin in the immunocomplexes was extracted using Magna ChIP A/G Kits (Millipores). Specimens were Proteinase $\mathrm{K}$ treated to remove protein residues and condensed through spin columns. Whole genome sequencing and $2 \times 150$ paired-end reads was performed using an Illumina HiSeq4000 system (Illumina, Inc.). CLC Genomics Workbench (v10.) and Transcription Factor ChIP-Seq analysis pipeline were utilized for quality control (>20M reads), trimming (average length of reads $<150 \mathrm{bp}$ ), mapping (mouse genome build $38 / \mathrm{mm} 10$, total mapped reads $>20 \mathrm{M}$ ), and peak characterization (peak shape score, and p-value). Fold changes of RPM for peak position between KO and WT groups were analyzed using DESeq software (R package version 1.16.0). Heatmap of read peaks annotating transcription start site (TSS) within upstream and downstream $5 \mathrm{~kb}$ were characterized using gplots $\mathrm{R}$ package (v. 2.17.0, https://CRAN.R-project.org/package=gplots). BAM files were also visualized by the Integrative Genomics Viewer (v.2.4.13) and imported to SeqMock (v.1.42.0) with pair distance cutoff $200 \mathrm{bp}$. Data are available at GEO database (accession number GSE \#121698). Genome-wide expression profiles and ontology of aligned gene set were verified using gene set enrichment analysis (GSEA) ${ }^{69}$ and KEGG database.

\section{ChIP-PCR assessment of H3K27me3 enrichment in promoter regions}

Aliquots of $0.1 \mathrm{ng}$ DNA isolated from H3K27me3 immunoprecipates were subjected to probing the sequences of Sox9, Igf-2, and Wnt10a promoters proximal to transcription start site using specific Cy3conjugated primers (Applied Biosystems) (Supplementary Table 1) and ABI 7900 Detection System. Ct 
values for serial 10-fold dilution of DNA were detected to verify amplification efficiency for PCR analysis of each gene. H3K27me3 occupancy in promoters of interest was expressed as \% input.

\section{Statistical analyses}

Student's t-test was employed to analyze the difference between clinical OA and non-OA cartilage of specimens. Wilcoxon test was utilized to verify the difference between WT and KO mice. ANOVA followed by Bonferroni post-hoc test was used to delineate difference between different groups of experimental animals or articular chondrocyte cultures. Significant difference was defined as $p$ values $<0.05$.

\section{Abbreviations}

H3K27me2/me3, di-/trimethylated lysine 27 of histone 3; EZH2, enhancer of zeste homolog 2; EED, embryonic ectoderm development; SUZ12, polycomb repression complex 2 subunit, JMJD3, jumonji domain containing 3; IGF-2, insulin-like growth factor-2; OA, osteoarthrisis; ECM, extracellular matrix; ACh, articular chondrocyte; AC, articular cartilage; DOTIL, disruptor of telomeric silencing 1-like; ChIP-PCR, chromatin immunoprecipitation-PCR; BMD, bone mineral density; BV/TV, bone volume/total volume; WT, wildtype; KO, knockout; IL-1 $\beta$, interleukin-1 $\beta$; ChIP-seq, chromatin immunoprecipitation-sequencing; TSS, transcriptional start sites.

\section{Declarations}

\section{Acknowledgements}

This work was in part supported by grants [NHRI-EX110-1102SI] from National Health Research Institute; [MOST 107-2314-B-182A-038-MY3; MOST109-2628-B-182A-008] form Ministry of Science and Technology, and [CMRPG8K0041-3] from Chang Gung Memorial Hospital, Taiwan.

\section{Author contributions}

Study conception and design: WS Lian, RW Wu, JY Ko, YS Chen, SY Wang, CP Yu, H Jahr, FS Wang Acquisition of data: RW Wu, JY Ko diagnosed knee OA in patients and experimental animals; WS Lian performed ChIP-seq assays and histomorphometry; YS Chen performed mCT analysis, cell cultures, RTPCR, ChIP-PCR, genotyping \& gene construction; SY Wang performed experimental OA study, gait analysis \& immunoblotting; CP Yu performed epigenomic bioinformatics analysis.

Analysis and interpretation of data: WS Lian, RW Wu, JY Ko, H Jahr, FS Wang

Article drafting and revising: WS Lian, RW Wu, JY Ko, H Jahr, FS Wang

Article drafting and revising: WS Lian, JY Ko, H Jahr, FS Wang

\section{Competing financial interests}


WS Lian, RW Wu, JY Ko, YS Chen, SY Wang, CP Yu, H Jahr, and FS Wang have no financial competing interest.

\section{References}

1. Sharma, L. Osteoarthritis of the Knee. Engl. J. Med. 384, 51-59 (2021).

2. Jeon, O. et al. Local clearance of senescent cells attenuates the development of posttraumatic osteoarthritis and creates a pro-regenerative environment. Med. 23, 775-781 (2017).

3. Monteagudo, S. \& Lories, R. J. Cushioning the cartilage: a canonical Wnt restricting matter. Rev. Rheumatol.13, 670-681 (2017).

4. Sugita, S. et al. Transcription factor Hes 1 modulates osteoarthritis development in cooperation with calcium/calmodulin-dependent protein kinase 2. Natl. Acad. Sci. U. S. A.112, 3080-3085 (2015).

5. Kim, J. H. et al. Regulation of the catabolic cascade in osteoarthritis by the zinc-ZIP8-MTF1 axis. Cell 156, 730-743 (2014).

6. Sen, P., Shah, P. P., Nativio, R. \& Berger, S. L. Epigenetic mechanisms of longevity and aging. Cell 166, 822-839 (2016).

7. Fang, D. et al. The histone H3.3K36M mutation reprograms the epigenome of chondroblastomas. Science 352, 1344-1348 (2016).

8. Hata, K. et al. Arid5b facilitates chondrogenesis by recruiting the histone demethylase Phf2 to Sox9regulated genes. Commun.4, 2850 (2013).

9. Zhang, M. et al. Epigenetically mediated spontaneous reduction of NFAT1 expression causes imbalanced metabolic activities of articular chondrocytes in aged mice. Osteoarthritis Cartilage 24, 1274-1283 (2016).

10. Monteagudo, S. et al. DOT1L safeguards cartilage homeostasis and protects against osteoarthritis. Commun.8,15889 (2017).

11. Wang, S. P. et al. UTX-MLL4-p300 transcriptional regulatory network coordinately shapes active enhancer landscapes for eliciting transcription. Cel/67, 308-321 (2017).

12. Tran, N., Broun, A. \& Ge, K. Lysine demethylase KDM6A in differentiation, development, and cancer. Cell Biol. 40, e00341-20 (2020).

13. Kim, K. I., Park, Y. S. \& Im, G. I. Changes in the epigenetic status of the SOX-9 promoter in human osteoarthritic cartilage. Bone Miner. Res.28, 1050-1060 (2013).

14. Lui, J. C. et al. EZH1 and EZH2 promote skeletal growth by repressing inhibitors of chondrocyte proliferation and hypertrophy. Commun.7, 13685 (2016).

15. Du, X. et al. Ezh2 ameliorates osteoarthritis by activating TNFSF13B. J. Bone Miner. Res. 35, 956-965 (2020).

16. Mirzamohammadi, F. et al. Polycomb repressive complex 2 regulates skeletal growth by suppressing Wnt and TGF- $\beta$ signalling. Commun. 7, 12047 (2016). 
17. Yapp, C., Carr, A. J., Price, A., Oppermann, U. \& Snelling, S. J. H3K27me3 demethylases regulate in vitro chondrogenesis and chondrocyte activity in osteoarthritis. Arthritis Res. Ther. 18, 158 (2016).

18. Wang, C. et al. UTX regulates mesoderm differentiation of embryonic stem cells independent of H3K27 demethylase activity. Natl. Acad. Soc. U. S. A. 109, 15324-15328 (2012).

19. Ko, J. Y., Sun, Y. C., Li, W. C. \& Wang, F. S. Chaperonin 60 regulation of SOX9 ubiquitination mitigates the development of knee osteoarthritis. Mol. Med. 94, 755-769 (2016).

20. Lorenz, J. \& Grässel, S. Experimental osteoarthritis models in mice. Methods Mol. Biol. 1194, 401-419 (2014).

21. Hunter, D. J. \& Bierma-Zeinstra. S. Osteoarthritis. Lancet. 393, 1745-1759 (2019).

22. Ruan, M. Z., Petel, R. M., Dawson, B. C., Jiang, M. M. \& Lee, B. H. Pain, motor and gait assessment of osteoarthritis in a cruciate ligament transection model. Osteoarthritis Cartilage 21, 1355-1364 (2013).

23. Baron, J. et al. Short and tall stature: a new paradigm emerges. Rev. Endocrinol.11, 735-746 (2015).

24. Monteagudo, S. \& Lories, R. J. Cushioning the cartilage: a canonical Wnt restricting matter. Rev. Rheumatol.13, 670-681 (2017).

25. McCulloch, K., Litherland, G. J. \& Rai, T. S. Cellular senescence in osteoarthritis pathology. Aging Cell 16, 210-218 (2017).

26. Frank-Bertoncelj, M. et al. Epigenetically-driven anatomical diversity of synovial fibroblasts guides joint-specific fibroblasts functions. Commun. 8, 14852 (2017).

27. Steck, E., Bräun, J., Pelttari, K., Kadel, S., Kalbacher, H. \& Richter, W. Chondrocytes secretes CRTAC1: a glycosylated extracellular matrix molecule of human articular cartilage. Matrix Biol. 26, 30-41 (2007).

28. Etich, J., Holzer, T., Pitzler, L., Bluhm, B. \& Brachvogel, B. MiR-26a modulates extracellular matrix homeostasis in cartilage. Matrix Biol. 43, 27-34 (2015).

29. Yasuda, H., Oh, C. D., Chen, D., de Crombrugghe, B. \& Kim, J. H. A novel regulatory mechanism of type II collagen expression via a SOX9-dependent enhancer in intron 6. Biol. Chem. 292, 528-538 (2017).

30. Kruidenier, L. et al. A selective jumonji H3K27 demethylase inhibitor modulates the proinflammatory macrophage response. Nature 488, 404-408 (2012).

31. Leijten, J. C. et al. GREM1, FRZB and DKK1 mRNA levels correlate with osteoarthritis and are regulated by osteoarthritis-associated factors. Arthritis Res. Ther. 15, R126 (2013).

32. Deng, Z. H., Li, Y. S., Gao, X., Lei, G. H. \& Huard, J. Bone morphogenetic proteins for articular cartilage regeneration. Osteoarthritis Cartilage. 26, 1153-1161 (2018)

33. Vincent, T. L. Of mice and men: converging on a common molecular understanding of osteoarthritis. Lancet Rheumatol. 2, e633-e645 (2020).

34. Dunn, S. L., Soul, J., Anand, S., Schwartz, J. M., Boot-Handford, R. P. \& Hardingham, T. E. Gene expression changes in damaged osteoarthritic cartilage identify a signature of non-chondrogenic and mechanical responses. Osteoarthritis Cartilage. 24, 1431-40 (2016).

35. Grogan, S. P., Miyaki, S., Asahara, H., D'Lima, D. D. \& Lotz, M. K. Mesenchymal progenitor cell markers in human articular cartilage: normal distribution and changes in osteoarthritis. Arthritis Res. Ther. 11, 
R85 (2009).

36. Vincent, T. L. \& Wann, A. K. T. Mechanoadaptation: articular cartilage through thick and thin. Physiol. 597, 1271-1281 (2019).

37. Charlier, E. et al. Chondrocyte differentiation and osteoarthritis (OA). Pharmacol. 165, 49-65 (2019).

38. Zhan, T. et al. MEK inhibitors activate Wnt signaling and induce stem cell plasticity in colorectal cancer. Commun. 10, 2197 (2019).

39. Katz, J. N., Arant, K. R. \& Loeser, R. F. Diagnosis and treatment of hip and knee osteoarthritis: a review. JAMA 325, 568-578 (2021)

40. Latourte, A., Kloppenburg, M. \& Richette P. Emerging pharmaceutical therapies for osteoarthritis. Rev. Rheumatol. 16, 673-688 (2020).

41. Doolittle, M. L. et al. Genetic analysis of osteoblast activity identifies Zbtb40 as a regulator of osteoblast activity and bone mass. Genet. 16, e1008805 (2020).

42. Mason, J. B. et al. Wnt10b and Dkk-1 gene therapy differentially influenced trabecular bone architecture, soft tissue integrity, and osteophytosis in a skeletally mature rat model of osteoarthritis. Tissue Res. 58, 542-552 (2017).

43. Varela-Eirin, M. et al. Cartilage regeneration and ageing: Targeting cellular plasticity in osteoarthritis. Ageing Res. Rev. 42, 56-71 (2018).

44. Borensztein, M. et al. Contribution of epigenetic landscapes and transcription factors to Xchromosome reactivation in the inner cell mass. Commun.8, 1297 (2017).

45. Lietman, C. et al. Inhibition of Wnt/ $\beta$-catenin signaling ameliorates osteoarthritis in a murine model of experimental osteoarthritis. JCl Insight 3, e96308 (2018).

46. Clevers, H. \& Nusse, R. Wnt/ $\beta$-catenin signaling and disease. Cell 149, 1192-1205 (2012).

47. Uchimura, T. et al. An essential role for IGF2 in cartilage development and glucose metabolism during postnatal long bone growth. Development 144, 3533-3546 (2017).

48. Uchimura, T., Foote, A. T., Smith, E. L., Matzkin, E. G. \& Zeng, L. Insulin-like growth factor II (IGF-II) inhibits IL-1 $\beta$-induced cartilage matrix loss and promotes cartilage integrity in experimental osteoarthritis. Cell. Biochem. 116, 2858-2869 (2015).

49. Jin, C. et al. Histone demethylase UTX-1 regulates C. elegans life span by targeting the insulin/IGF-1 signaling pathway. Cell Metab. 14, 161-172 (2011).

50. Maures, T. J., Greer, E. L., Hauswirth, A. G. \& Brunet, A. The H3K27 demethylase UTX-1 regulates C. elegans life in a germline-independent insulin-dependent manner. Aging Cell. 10,980-990 (2011).

51. Zhang, L., Smith, D. W., Gardiner, B. S. \& Grodzinsky, A. J. Modeling the insulin-like growth factor system in articular cartilage. PLoS One. 8, e66870 (2013).

52. Hunter, D. J. \& Bierma-Zwinstra, S. Osteoarthritis. Lancet 393, 1745-1759 (2019).

53. Xu, Y., Tian, N. X., Bai, Q. Y., Chen, Q., Sun, X. H. \& Wang, Y. Gait assessment of pain and analgesics: comparison of the DigiGait ${ }^{\mathrm{TM}}$ and CatWalk ${ }^{\mathrm{TM}}$ gait imaging system. Bull. 35, 401-418 (2019). 
54. Lan, F. et al. A histone H3 lysine 27 demethylase regulates animal posterior development. Nature 449, 689-694 (2007).

55. Ler, L. D. et al. Loss of tumor suppressor KDM6A amplifies PRC2-regulated transcriptional repression in bladder cancer and can be targeted through inhibition of EZH2. Transl. Med. 9, 378 (2017).

56. Moody, J. D. et al. First critical repressive H3K27me3 marks in embryonic stem cells identified using designed protein inhibitor. Natl. Acad. Sci. U. S. A.114, 10125-10130 (2017).

57. van der Meulen, J., Speleman, F. \& van Vlierberghe, P. The H3K27me3 demethylase UTX in normal development and disease. Epigenetics 9, 658-668 (2014).

58. Lee, M. G. et al. Demethylation of H3K27 regulates polycomb recruitment and H2A ubiquitination. Science. 318, 447-50 (2007).

59. Laugesen, A., Højfeldt, J. W. \& Helin, K. Molecular mechanisms directing PRC2 recruitment and H3K27 methylation. Cell. 74, 8-18 (2019)

60. Hemming, S. et al. EZH2 deletion in early mesenchyme compromises postnatal bone microarchitecture and structural integrity and accelerates remodeling. FASEB J. 31, 1011-1027 (2017).

61. Di Croce, L. \& Helin, K. Transcriptional regulation by Polycomb group proteins. Struct. Mol. Biol. 20, 1147-1155 (2013).

62. Shan, Y. et al. PRC2 specifies ectoderm lineages and maintains pluripotency in primed but naïve ESCs. Commun. 8, 672 (2017).

63. De Santa, F., Totaro, M. G., Prosperini, E., Notarbartolo, S., Testa, G. \& Natoli, G. The histone H3 lysine27 demethylase Jmjd3 links inflammation to inhibition of polycomb-mediated gene silencing. Cell 130, 1083-1094 (2007).

64. Gosset, M., Berenbaum, F., Thirion, S. \& Jacques, C. Primary culture and phenotyping of murine chondrocytes. Proc. 3, 1253-1260 (2008).

65. Jia, H., Ma, X., Tong, W., Doyran, B., Sun, Z., Wang, L. et al. EGFR signaling is critical for maintaining the superficial layer of articular cartilage and preventing osteoarthritis initiation. Nat. Acad. Sci. U. S. A. 113, 14360-14365 (2016).

66. Nomura, M., Sakitani, N., Iwasawa, I., Kohara, Y., Takano, S., Wakimoto, Y. et al. Thinning of articular cartilage after joint unloading or immobilization. An experimental investigation of the pathogenesis in mice. Osteoarthritis Cartilage 25, 727-736 (2017).

67. Glasson, S. et al. The OARSI histopathology initiative-recommendations for histological assessment of osteoarthritis in the mouse. Osteoarthritis Cartilage 18, S17-23 (2010).

68. Lian W. S. et al. MicroRNA-128a represses chondrocyte autophagy and exacerbates knee osteoarthritis by disrupting Atg12. Cell Death Dis. 9, 919 (2018).

69. Subramanian, A. et al. Gene set enrichment analysis: a knowledge-based approach for interpreting genome-wide expression profiles. Natl. Acad. Sci. U S. A. 102, 15545-15550 (2005). 


\section{Figures}

a

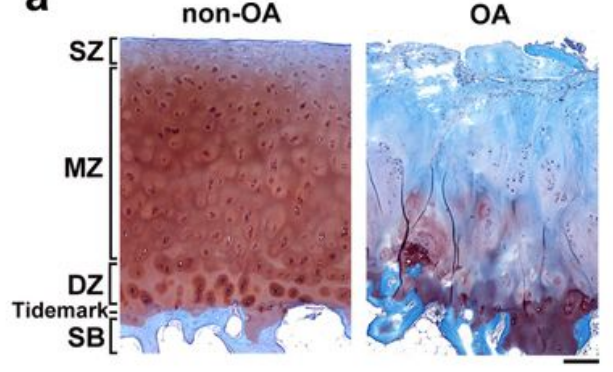

b

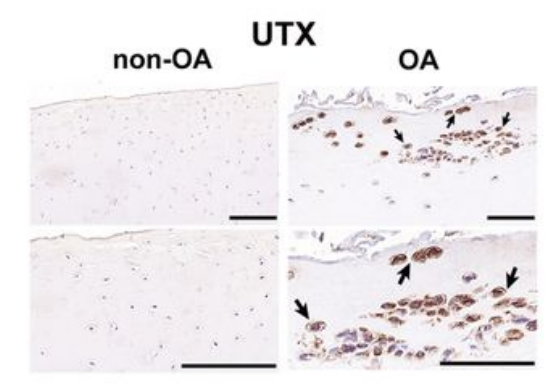

C

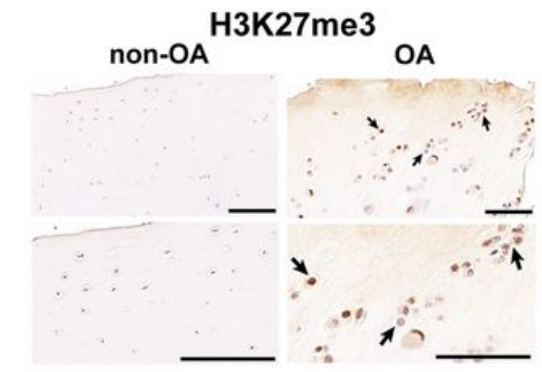

OnON-OA $\bullet$ OA
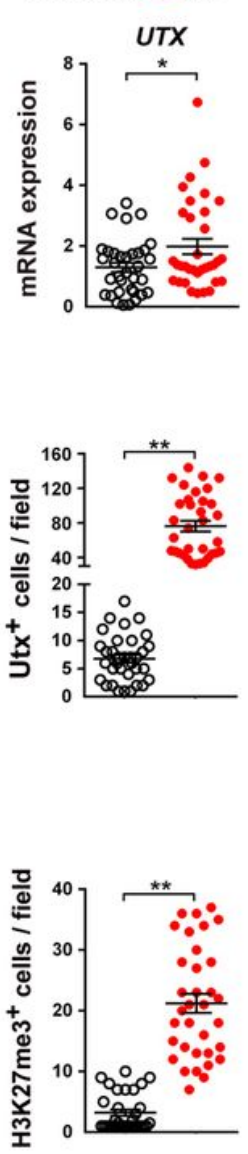

d
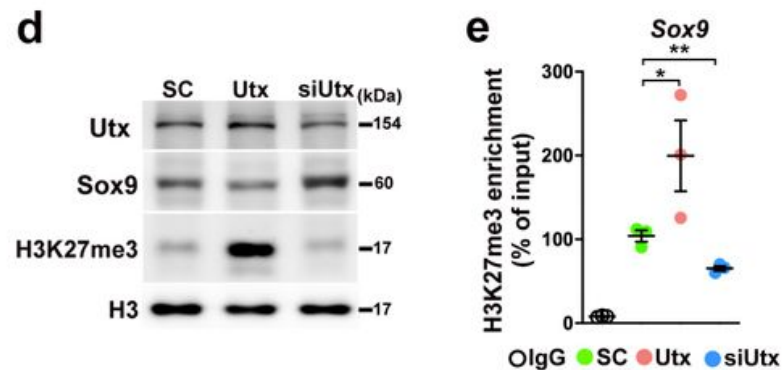

f

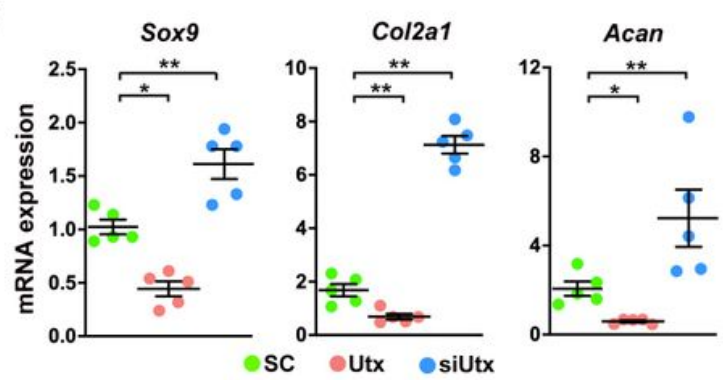

g

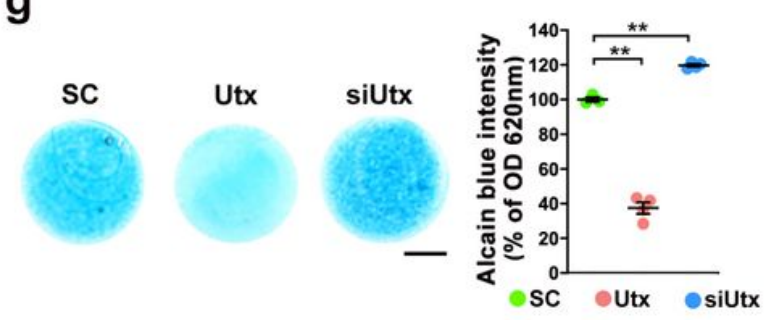

\section{Figure 1}

UTX-dependent reduction in chondrocytic activities. Safranin-O staining of macroscopically normal human articular cartilage (on left, non-OA) next to severely osteoarthritic tissue (OA). Quantification of UTX mRNA abundance in both tissues revealed elevated UTX expression in gonarthrotic cartilage (a); scale bar, $200 \mu \mathrm{m}$. Strong UTX (b) and H3K27me3 (c) immunostaining in osteoarthritic chondrocytes, next to quantified protein expression from 34 donors; scale bar, $20 \mu \mathrm{m}$ (low magnification), $10 \mu \mathrm{m}$ (high magnification). Forced UTX expression (d) increased H3K27me3 levels and reduced Sox9 abundance of chondrocytic cells. Upregulated H3K27me3 enrichment in Sox9 promoter region (e), but reduced Sox9, Col2a1 and Acan expression (f), respectively, and glycosaminoglycan synthesis ( $g$ ) quantified by Alcian blue staining; scale bar, $500 \mu \mathrm{m}$. Culture experiments were repeated 4 times and data are expressed as mean \pm standard error; *, $\mathrm{p}<0.05$; ${ }^{* *}, \mathrm{p}<0.001$. SC, scrambled control; siUtx, Utx RNAi. 
a

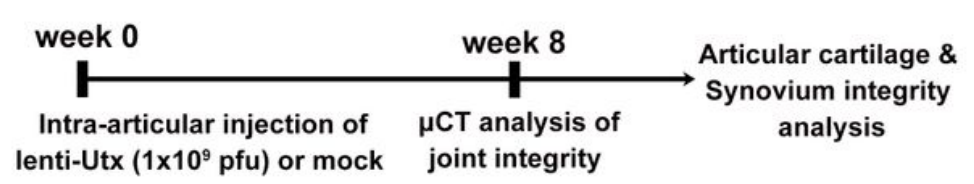

C

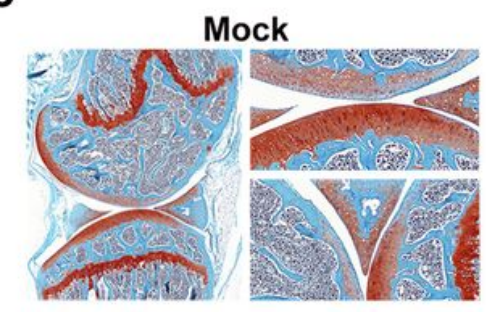

e

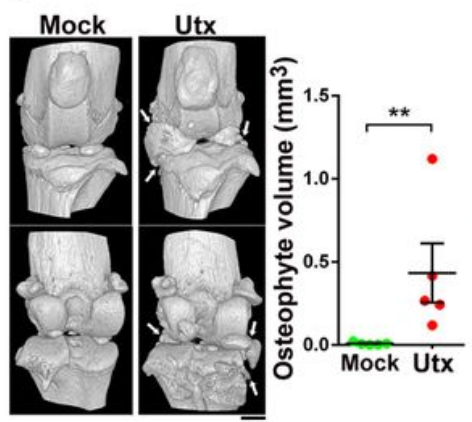

f

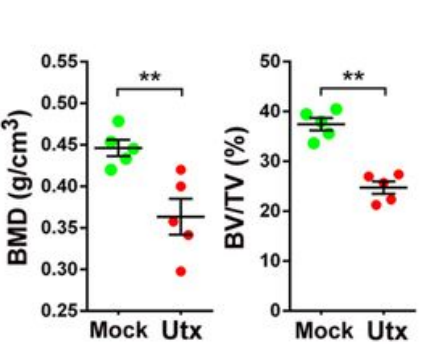

g b

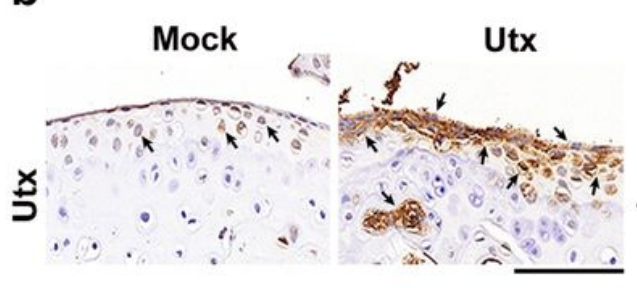

d
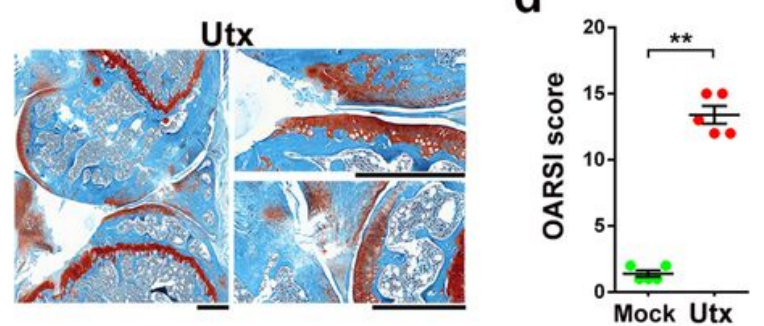
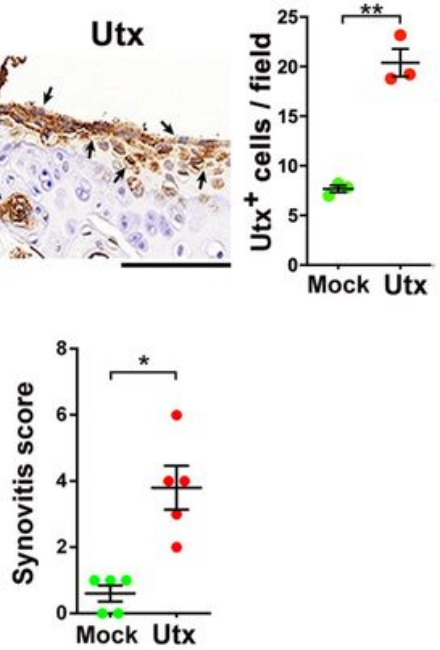

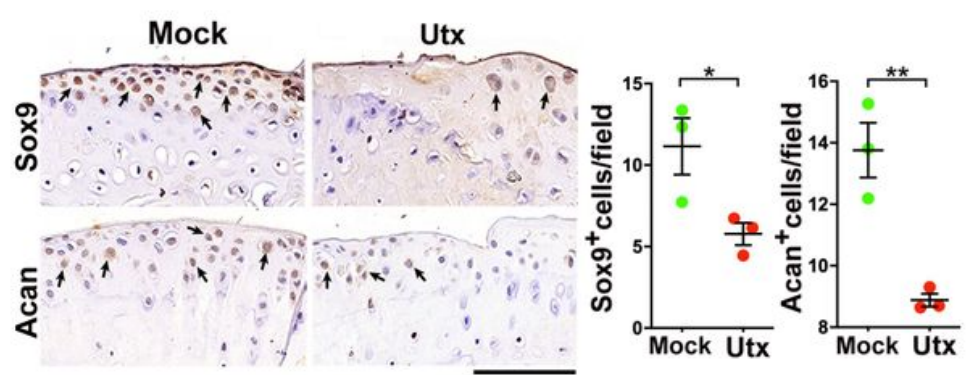

Figure 2

Signs of OA upon forced expression of Utx in mice. Schematic drawing for intra-artcular injection of lentivirus Utx (a). Strong Utx immunostaining in articular cartilage upon intra-articular injection with lentiviruses expressing Utx (b); scale bar, $10 \mu \mathrm{m}$. Severe cartilage disintegration and synovial hypertrophy in Utx-treated murine knees (c), as evident from OARSI and synovitis scoring (d); scale bar, $500 \mu \mathrm{m}$ (left image), $20 \mu \mathrm{m}$ (upper right image), and $100 \mu \mathrm{m}$ (lower right image). Forced Utx expression induced osteophyte formation (arrows) (e) but reduced BMD and BV/TV (f) of subchondral bone; scale bar, 0.5 $\mathrm{mm}$. Weak Sox9 and Acan immunoreactivity upon Utx treatment (g); scale bar, $10 \mu \mathrm{m}$. Data are expressed as mean \pm standard errors. * $p<0.05 ; * \star, p<0.001$. 


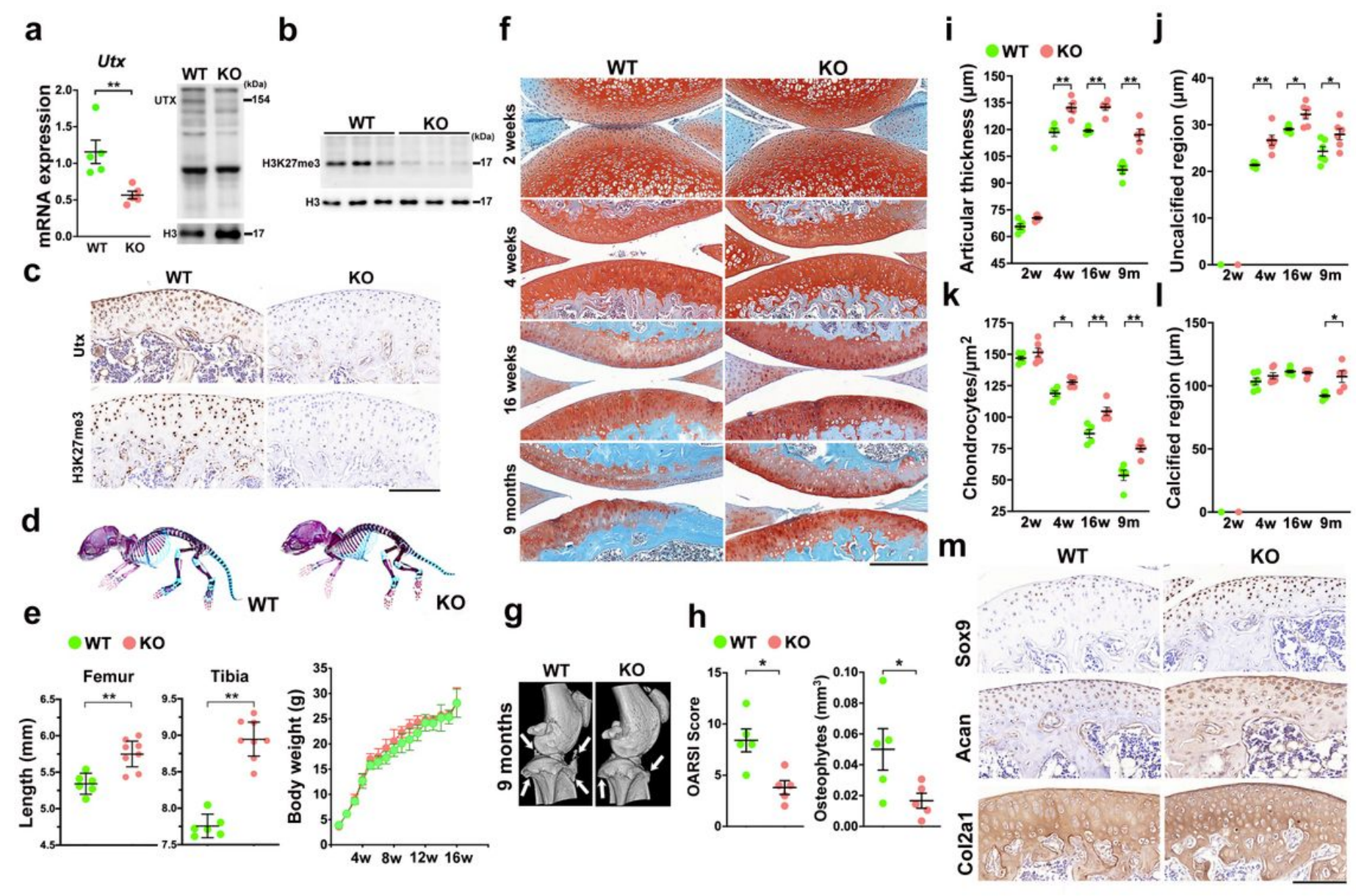

\section{Figure 3}

Utx knockout promotes ECM synthesis and protects articular cartilage integrity. Utx mRNA expression and protein abundance (a), respectively, confirming absence of Utx and reduced H3K27me3 levels (b) in KO mice. Cell culture experiments were performed 3 times. Data are expressed as mean \pm standard errors. Very faint Utx and H3K27me3 immunostaining in articular cartilage of KO mice (c); scale bar, $10 \mu \mathrm{m}$. Whole mount-stained skeleton in KO mice were like WT mice (d). Utx deletion did not significantly change body weight, but increased femur and tibia length (e). Safranin- 0 staining of articular cartilage (f; scale bar, $100 \mu \mathrm{m}$ ) and micro-CT images showing osteophyte formation (arrows) in 9-month-old KO and WT mice (g; scale bar, $0.5 \mathrm{~mm}$ ). Decreased OARSI scores and osteophyte volume in 9-month-old KO mice (h). Significantly increased relative thickness of articular cartilage (i), uncalcified (j) and density of articular chondrocytes $(k)$, respectively, and calcified articular cartilage regions $(\mathrm{I})$ in $\mathrm{KO}$ mice. Relatively stronger Sox9, Acan, and Col2a1 immunostaining $(\mathrm{m})$ in articular chondrocytes of 4-week-old Utx KO mice as compared to WT mice; scale bar, $10 \mu \mathrm{m}$. Data are expressed as mean \pm standard errors calculated from 6 mice. *, $p<0.05 ; * \star, p<0.001 ;$ n.s., no significance. 
a

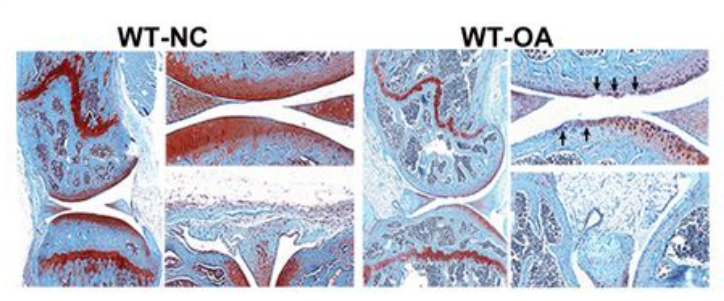

c
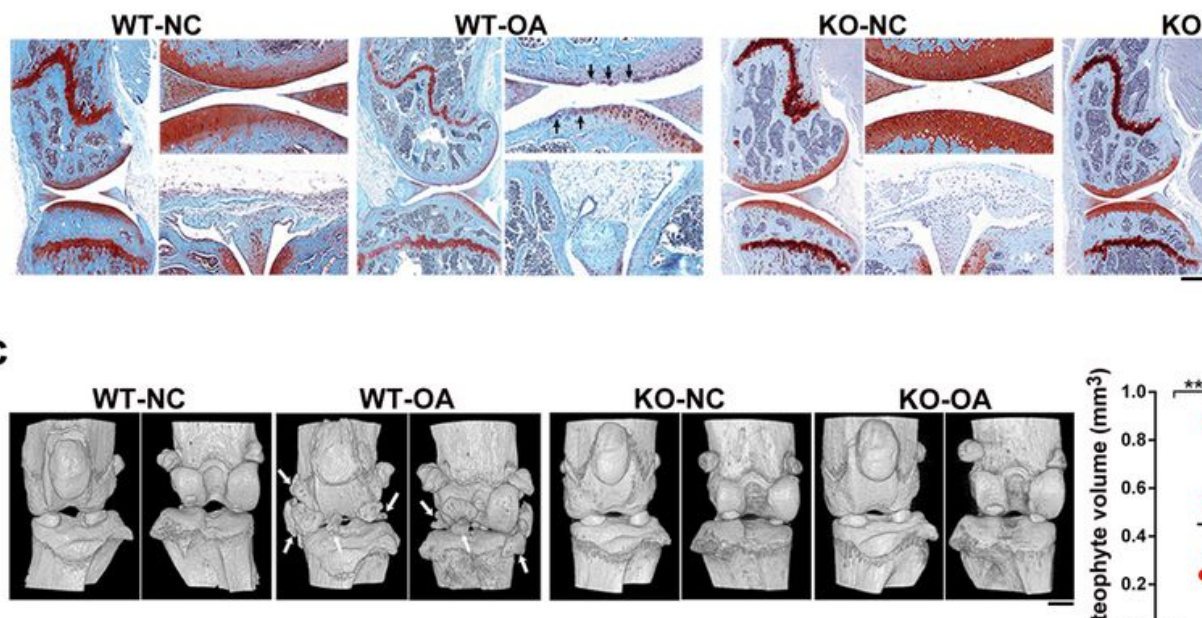

KO-OA

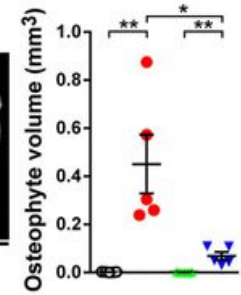

d

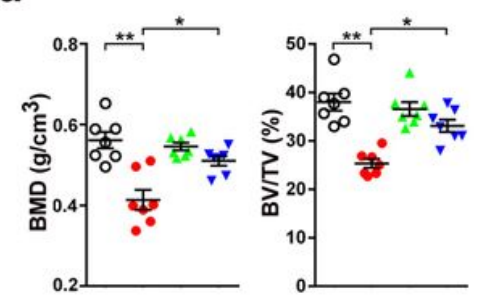

e

f

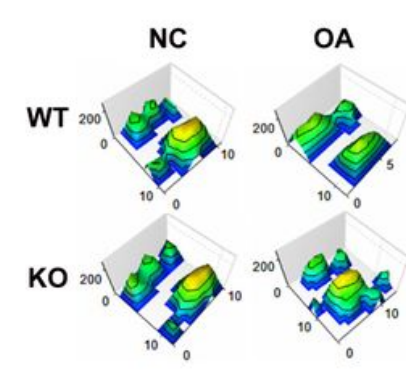

b

OWT $\odot W T+O A \triangle K O \quad$ KO+OA
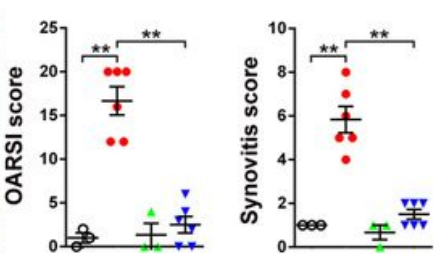

OWT $\bullet W T+O A \triangle K O \quad$ KO+OA

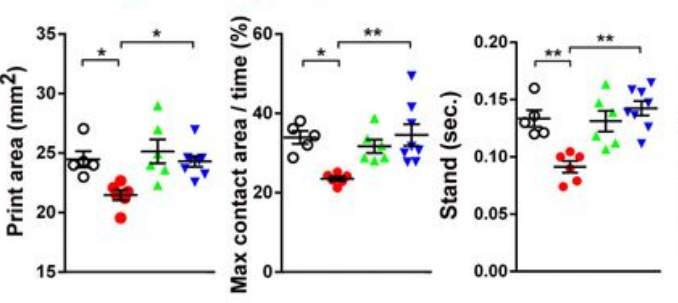

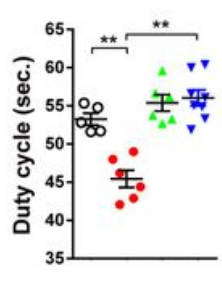

g

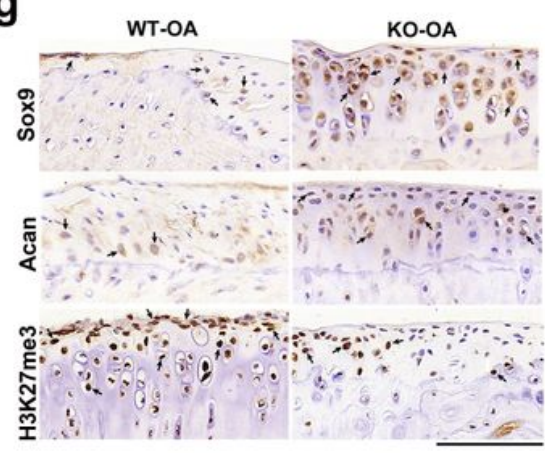

\section{Figure 4}

Utx KO in mice protects against gonarthritis. Intra-articular collagenase injections were used to induce OAlike symptoms (i.e. cartilage damage and synovitis) in murine knee joints. Animals received either collagenase (OA) or saline (NC) injections. Upon OA induction, 16-week-old Utx KO mice developed weaker symptoms than WT (WT-OA) animals as evident from Safranin-O staining (a) and quantitative OARSI and synovitis scores (b); scale bar, $500 \mu \mathrm{m}$ (left image), $20 \mu \mathrm{m}$ (upper right image), and $100 \mu \mathrm{m}$ (lower right image). Utx loss protected against osteophyte formation (c). Changes in bone mineral density (BMD) and subchondral bone volume fraction (BV/TV) of KO mice (d); scale bar, $500 \mu \mathrm{m}$. Footprint histograms (e) and gait profiles, including print area, maximum contact intensity, duration of sand and duty cycles ( $f$ ) suggest largely normal load-bearing of collagenase-injected joints in Utx KO mice as compared to limping WT animals. Prominent Sox9 and Acan immunostaining, but weak H3K27me3 immunoreactivity, in chondrocytes of collagenase-treated knees in $\mathrm{KO}$ mice (g); scale bar, $10 \mu \mathrm{m} . .{ }^{*}, \mathrm{p}<$ $0.05 ; * \star, p<0.001$. 
a

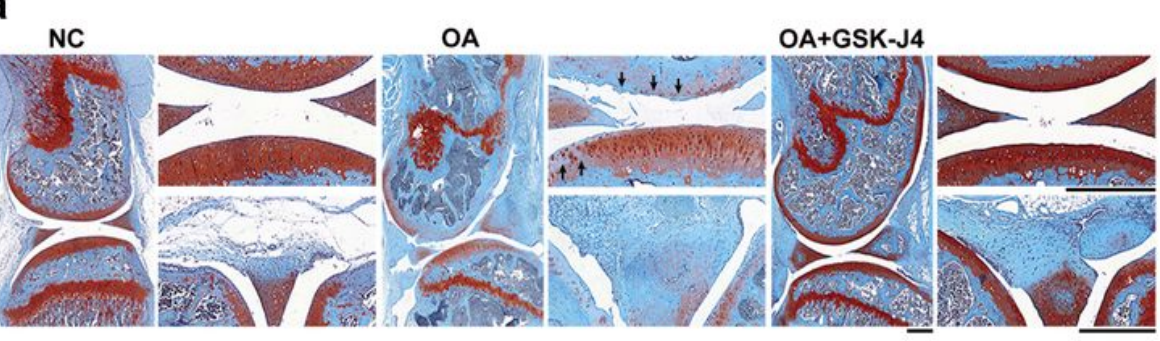

C

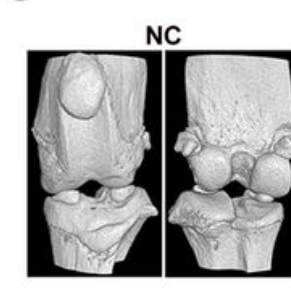

e

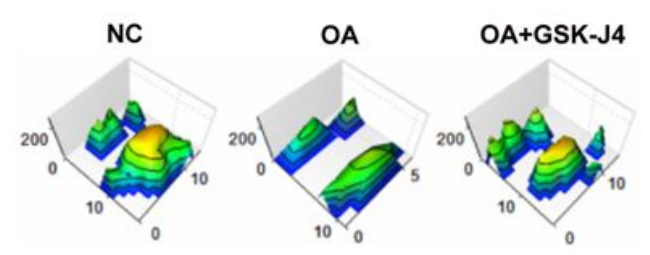

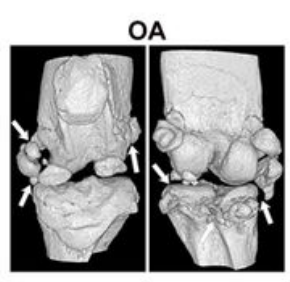

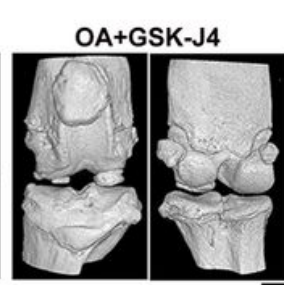

f

ONC $\bullet O A \triangle O A+G S K-J 4$

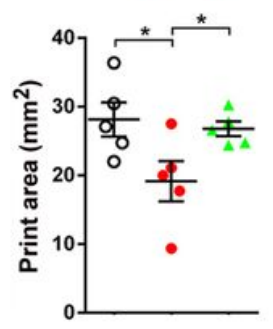

b

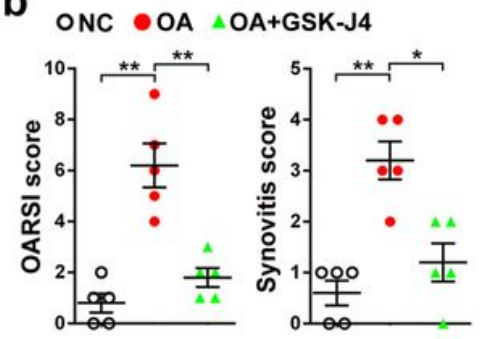

d

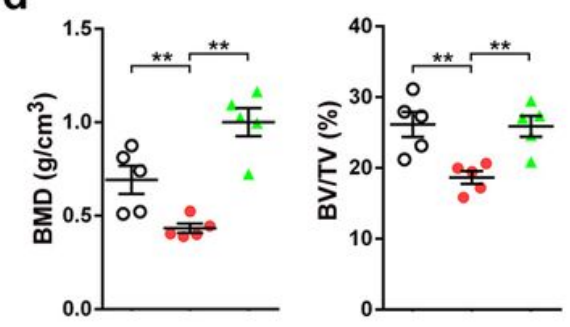

g

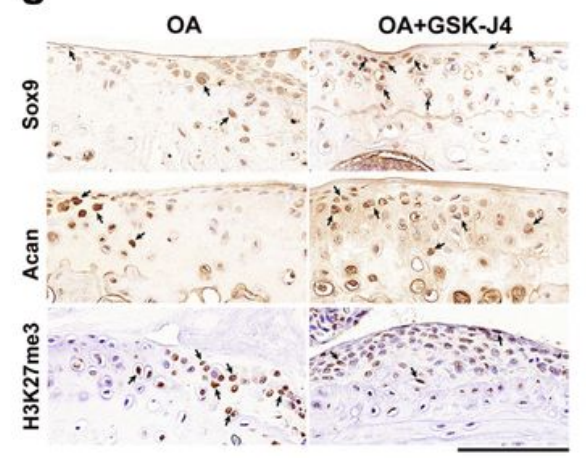

Figure 5

Pharmacological Utx inhibition protects against gonarthritis. Intra-articular treatment with GSK-J4 reduced collagenase-induced articular cartilage loss and synovial hyperplasia (a) together with decreases in OARSI and synovitis scores (b); scale bar, $500 \mu \mathrm{m}$ (left image), $20 \mu \mathrm{m}$ (upper right image), and $100 \mu \mathrm{m}$ (lower right image). GSK-J4 further reduced osteophyte formation (c) and improved subchondral BMD and BV/TV (d) of joints upon OA induction; scale bar, $500 \mu \mathrm{m}$. The administration improved footprint profiles (e) and print area and maximum contact duration in respective extremities (f), indicating pain alleviation in the affected joints. GSK-J4-treated joints showed prominent Sox9 and Acan immunostaining, but weak H3K27me3 immunoreactivity, in chondrocytes (g); scale bar, $10 \mu \mathrm{m} .$, NC. Vehicle; OA collagenase injection. 
a

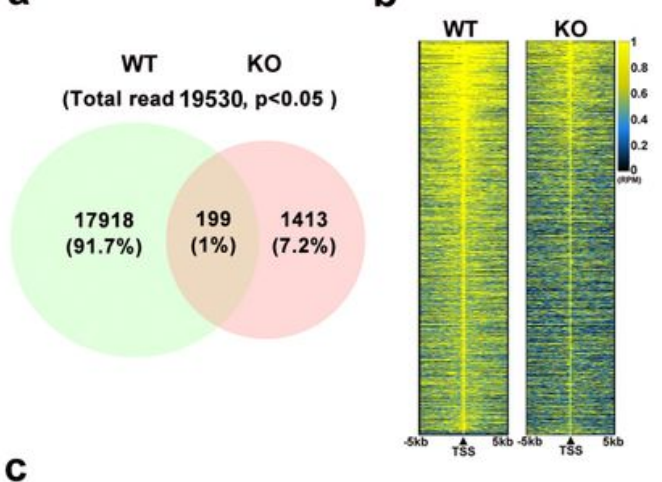

C

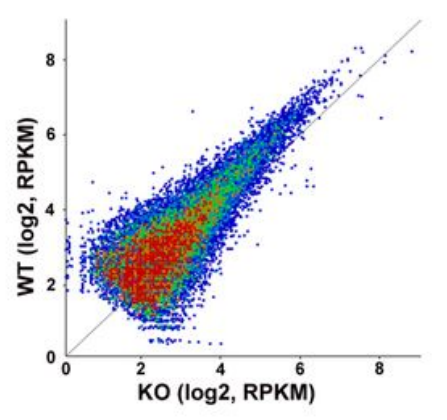

d

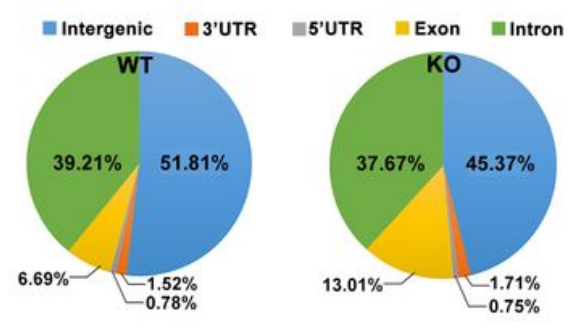

e

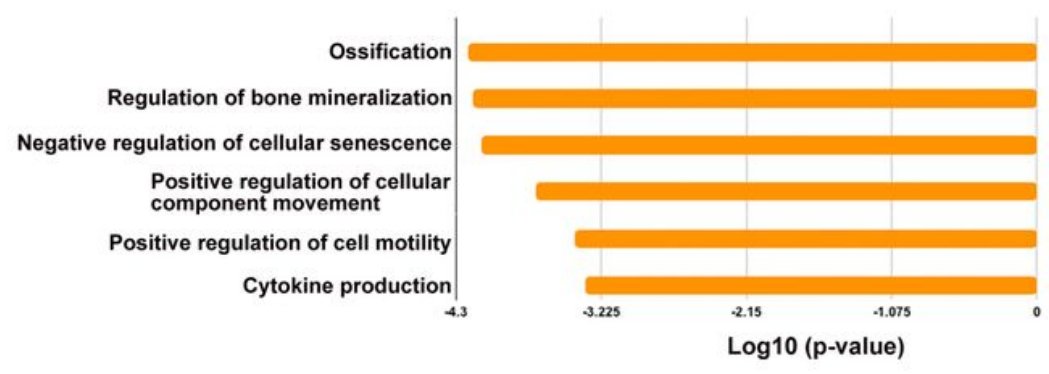

g

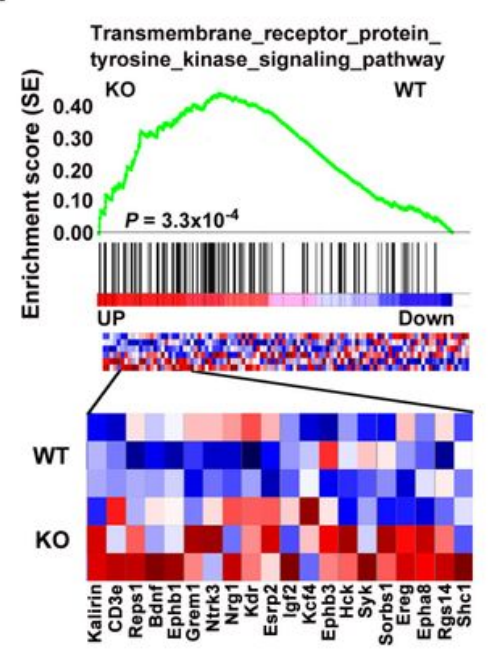

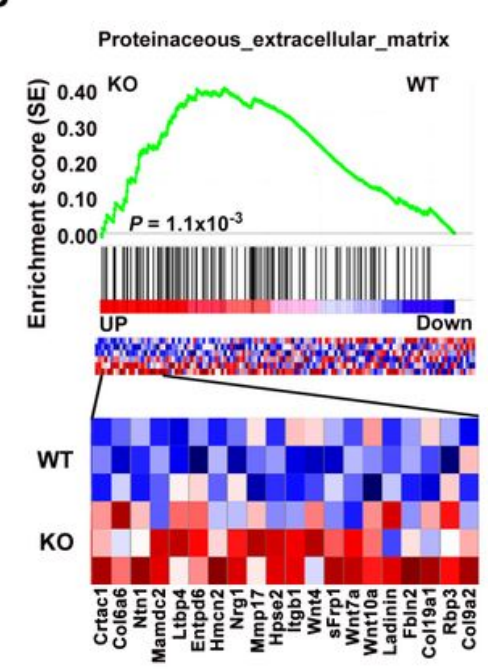

\section{Figure 6}

ChIP-seq analyses of H3K27me3 in chondrocytes. Venn diagram showing the overlap of H3K27me3 peaks between KO and WT mice. Total numbers of H3K27me3-enriched binding sites and their relative proportions (\%) are indicated. Note that KO mice revealed fewer binding sites compared to WT animals (a). Heatmaps of ChIP-seq analyses showing H3K27me3 DNA occupancy within 5 kb upstream and downstream, respectively, of transcriptional start site (TSS) (b). Scatter plots shows H3K27me3 ChIP-seq signals distribution in KO and WT (c), next to relative distribution of H3K27me3 occupancy across genomic regions in chondrocytes of WT and KO animal, respectively (d). Selected H3K27me3 enriched GO terms in KO mice (p-value<0.001) (e). Gene set enrichment analysis of H3K27me3 marks revealed significant enhancement two specific pathways in Utx KO cells. Igf-2 (f) and Wnt10a (g) were selected as differentially regulated and earlier reported H3K27me3-mediated candidates controlling chondrocyte differentiation (enlarged section). Data are calculated from 3 experiments. 
a
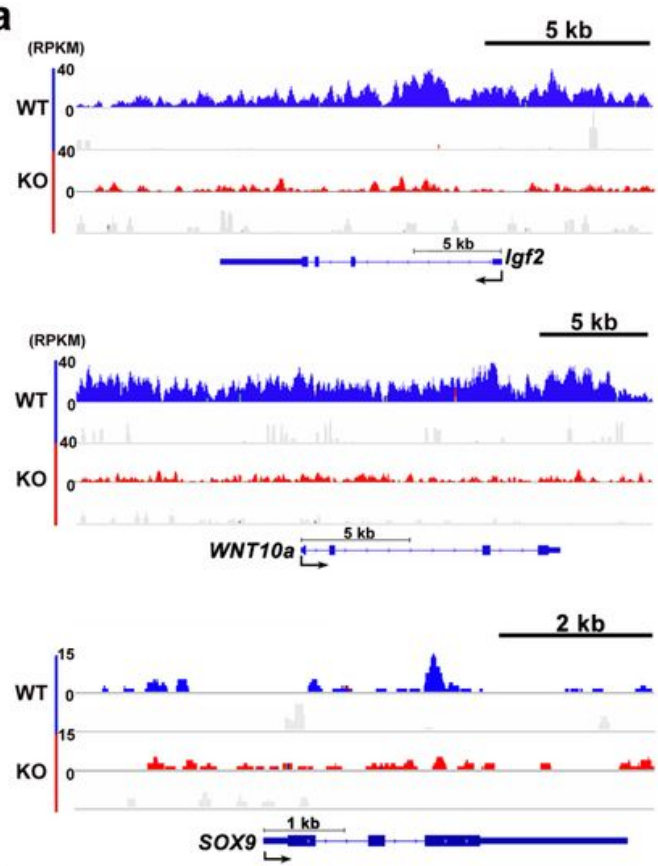

b

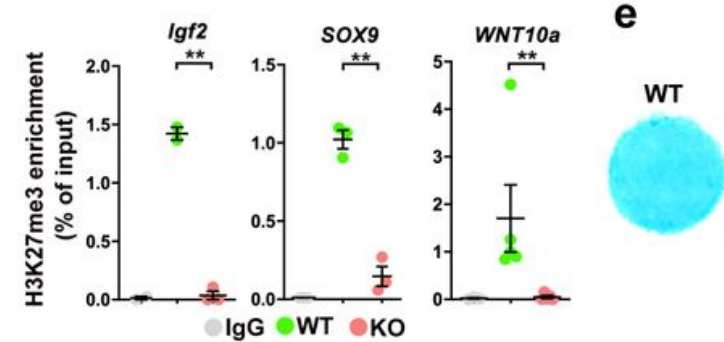

C

d
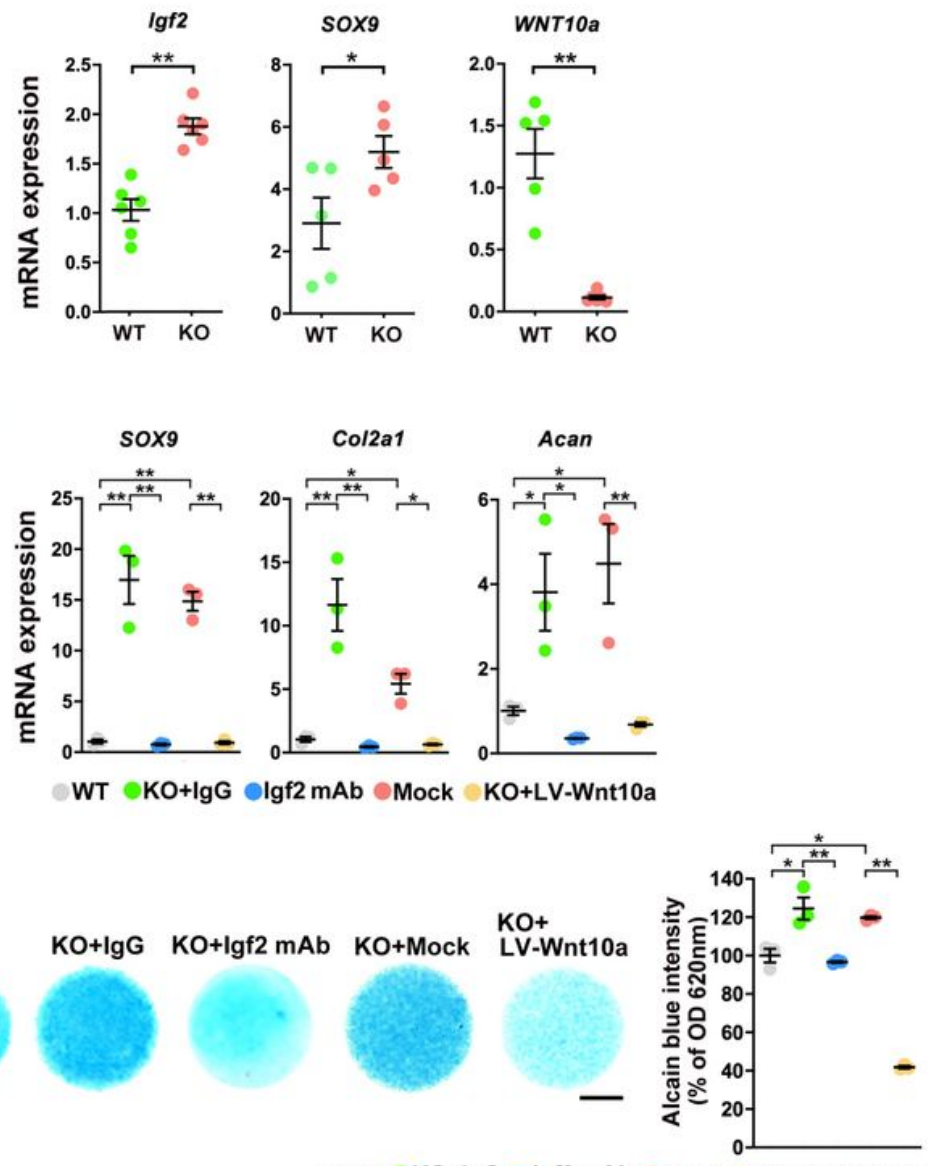

WT KO+lgG elgf2 mAb Mock KO+LV-Wnt10a

Figure 7

UTX-dependence of H3K27me3-mediated transcriptional repression. Gene track illustrating limited H3K27me3 chromatin occupation at regulator loci and promoter regions (a) of three selected genes (i.e., Igf-2, Wnt10a and master regulator Sox9) in Utx KO (red) as compared to WT chondrocytes (blue). This confirms highly significant differences in the enrichment of H3K27me3 by ChIP-PCR assay (b). Utx knockout increased transcription of Igf-2 and Sox9, but suppressed that of Wnt10a (c). Antibodymediated titration of Igf-2 and forced expression of Wnt10a both suppressed Sox9, Col2a1, and Acan expression (d), as well as glycosaminoglycan production (e), in Utx KO chondrocytes. Cell culture experiments were performed 3 times. Data are expressed as mean \pm standard errors. Scale bar, $500 \mu \mathrm{m}$. *, $p<0.05 ; * \star, p<0.001$. 
a

b

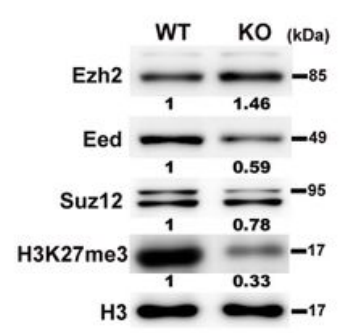

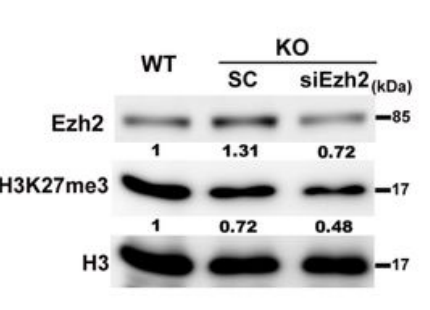

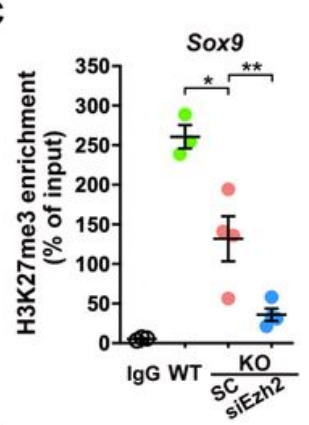

d

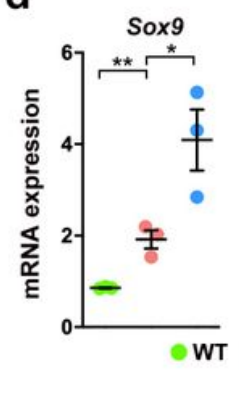

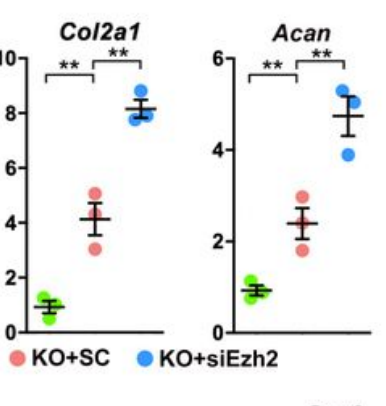

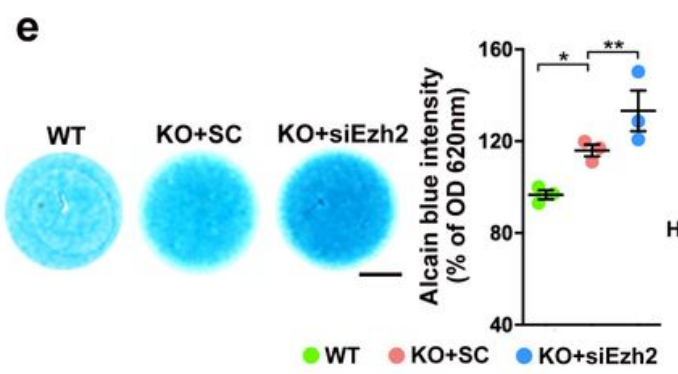

h

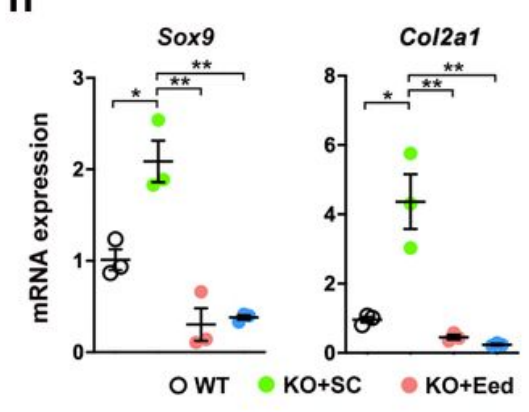

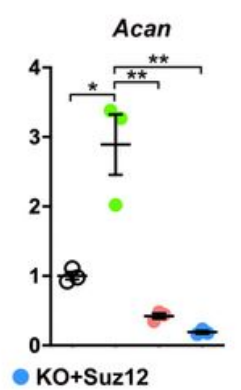

i
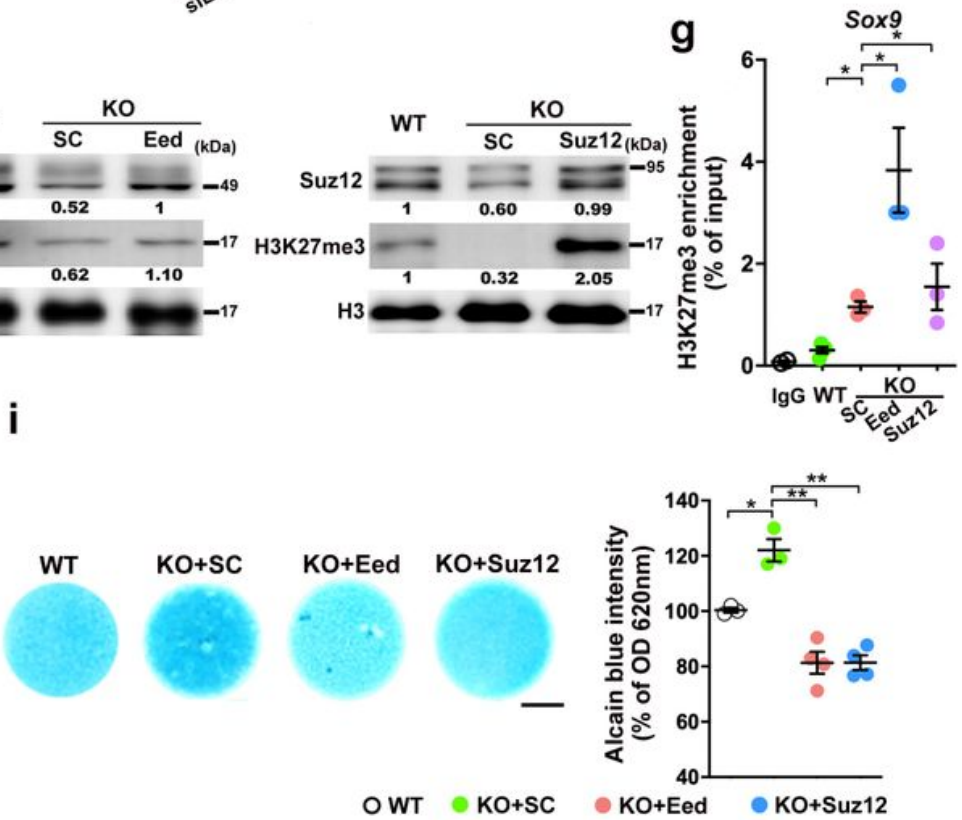

\section{Figure 8}

PRC2 core components contribute to Utx depletion-mediated anabolic chondrocytic activity. Loss of Utx function increased abundance of Ezh2, but reduced that of Eed and Suz12 (a). RNAi-mediated silencing of Ezh2 (siEzh2) reduced H3K27me3 levels (b) and its enrichment within Sox9 promoter regions of Utx KO chondrocytes (c). Ezh2 RNAi further increased Sox9, Col2a1, and Acan expression (d) and cartilage ECM synthesis (e). Forced Eed and Suz12 expression increased H3K27me3 abundance (f) and H3K27me3 occupancy at the Sox9 promoter (g). Chondrocytic maker gene expression (h) and ECM production (i) by chondrocytes from Utx KO mice were suppressed by forced expression of both components. Cell culture experiments were performed 3 times. Data are expressed as mean \pm standard errors. Scale bar, $500 \mu \mathrm{m} . *, p<0.05 ; * *, p<0.001$. 


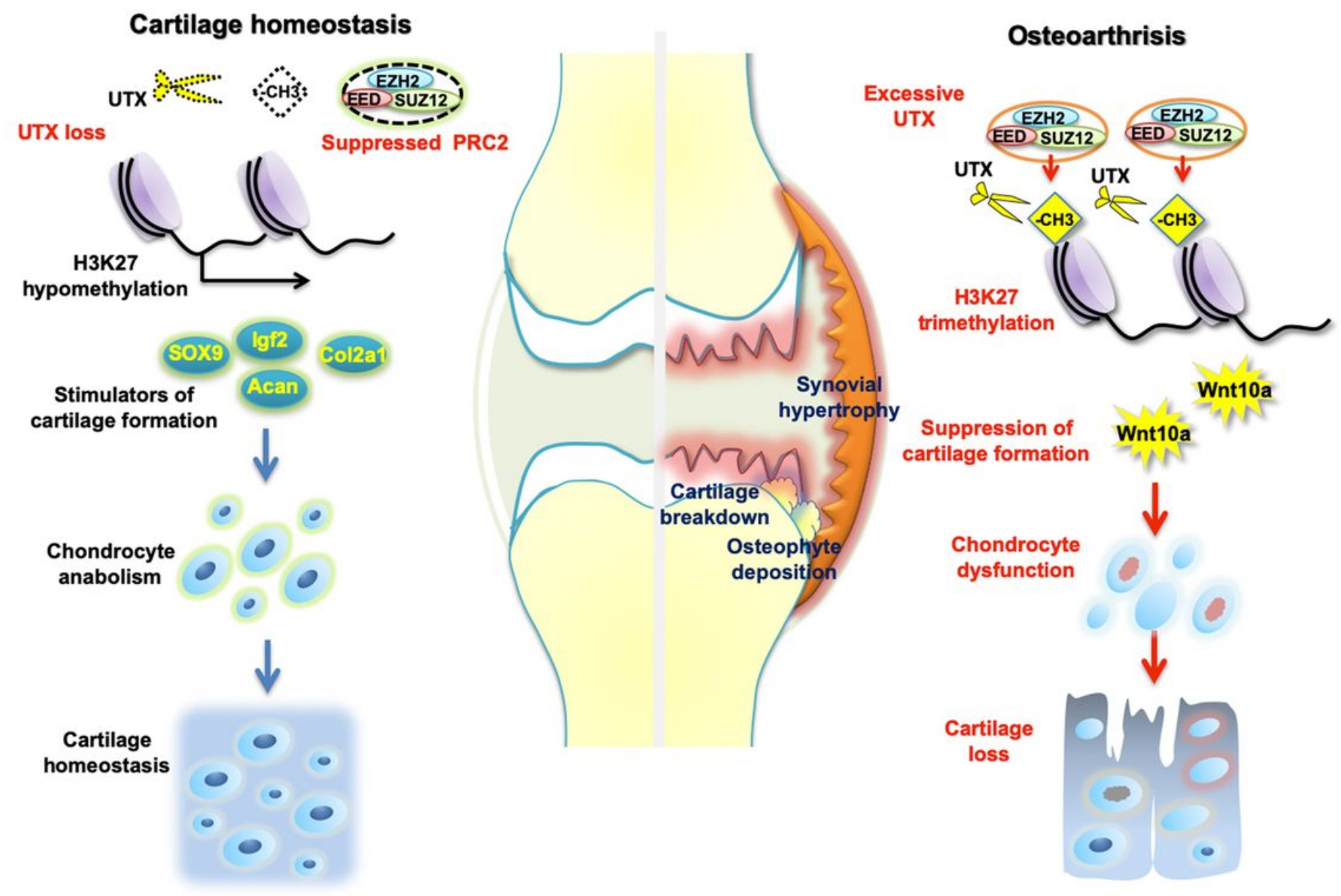

Figure 9

Schematic illustration of how epigenetic changes affect cartilage ECM integrity. Age-dependent changes in articular chondrocytes that are leading to an increased UTX activity (i.e., mimicked by lentiviral overexpression) are, together with histone writer PRC2 component EZH2, resulting in an elevated histone methylation state in these cells. This consequently dysregulates cartilage homeostasis through, among others, facilitated canonical Wnt10a signaling. Also, this epigenetic signature suppresses SOX9 activity, which is crucial for maintaining ECM integrity. Ultimately, this culminates in the development of OA (on left). In contrast, inhibiting UTX genetically or pharmacologically facilitates transcriptional activity at promoter regions of certain cartilage key markers (like e.g., SOX9, Col2a1, ACAN) at least partially through co-suppression of EZH2. This net stimulation of anabolic factors then aids in maintaining the ECM integrity of the tissue to ensure proper homeostasis (on right).

\section{Supplementary Files}

This is a list of supplementary files associated with this preprint. Click to download.

- SupplementaryTable1.docx 
- Figures1.tif

- Figures2.tif

- Figures3.tif

- Figures4.tif 